TRANSACTIONS OF THE

AMERICAN MATHEMATICAL SOCIETY

Volume 356, Number 9, Pages 3517-3543

S 0002-9947(03)03512-8

Article electronically published on November 25, 2003

\title{
PARABOLIC EVOLUTION EQUATIONS WITH ASYMPTOTICALLY AUTONOMOUS DELAY
}

\author{
ROLAND SCHNAUBELT
}

\begin{abstract}
We study retarded parabolic non-autonomous evolution equations whose coefficients converge as $t \rightarrow \infty$, such that the autonomous problem in the limit has an exponential dichotomy. Then the non-autonomous problem inherits the exponential dichotomy, and the solution of the inhomogeneous equation tends to the stationary solution at infinity. We use a generalized characteristic equation to deduce the exponential dichotomy and new representation formulas for the solution of the inhomogeneous equation.
\end{abstract}

\section{INTRODUCTION}

In the present paper we investigate the long-term behaviour of linear nonautonomous evolution equations

$$
\dot{u}(t)=A(t) u(t)+L(t) u_{t}+f(t), \quad t \geq s \geq 0, \quad u_{s}=\phi \in E,
$$

on a Banach space $X$. The equation is parabolic in so far the linear operators $A(t), t \geq 0$, are supposed to be sectorial of the same type and to satisfy the 'Acquistapace-Terreni' condition (see (P) in Section 2). Moreover, we let $r \geq 0$, $E:=C([-r, 0], X)$, and $u_{t}(\xi):=u(t+\xi)$ for $\xi \in[-r, 0], t \geq s$, and a function $u:[s-r, \infty) \rightarrow X$. Equation (1.1) also contains the retardation operators $L(t)$ : $E \rightarrow X, t \geq 0$, which are assumed to belong to $C_{b}\left(\mathbb{R}_{+}, \mathcal{L}_{s}(E, X)\right)$, the space of uniformly bounded, strongly continuous operator-valued functions. We finally require that the problem be asymptotically autonomous in the sense that there exist a sectorial operator $A$ and a bounded operator $L: E \rightarrow X$ such that

$$
\begin{aligned}
\lim _{t \rightarrow \infty} R(w, A(t)) & =R(w, A) \quad \text { in } \mathcal{L}(X), \\
\lim _{t \rightarrow \infty} L(t) & =L \quad \text { in } \quad \mathcal{L}\left(C^{\beta}([-r, 0], X), X\right)
\end{aligned}
$$

for some $\beta \in[0,1)$. This covers in particular the prototypical case

$$
L(t) \phi=B(t) \phi(-\tau(t)),
$$

where $\tau \in C\left(\mathbb{R}_{+},[-r, 0]\right)$ and $B(\cdot) \in C_{b}\left(\mathbb{R}_{+}, \mathcal{L}_{s}(X)\right)$ converge (in $\mathbb{R}$ and $\mathcal{L}(X)$, respectively) as $t \rightarrow \infty$. Observe that in (1.4) one does not have convergence in $\mathcal{L}(E, X)$.

Received by the editors January 18, 2002 and, in revised form, March 27, 2003.

2000 Mathematics Subject Classification. Primary 35R10; Secondary 34K30, 47D06.

Key words and phrases. Retarded parabolic evolution equation, asymptotically autonomous, exponential dichotomy, robustness, convergence of solutions, variation of parameters formula, characteristic equation, evolution semigroup. 
Let us first consider the undelayed case $L(t)=0$, i.e.,

$$
\dot{u}(t)=A(t) u(t)+f(t), \quad t>s \geq 0, \quad u(s)=x .
$$

It was established in [6 Thm. 6.2] (improving a closely related result in [32]) that the spectral condition

$$
\sigma(A) \cap i \mathbb{R}=\emptyset
$$

implies the exponential dichotomy (on a time interval $[a, \infty) \subseteq \mathbb{R}_{+}$) of the evolution family $U(\cdot, \cdot)$ solving (1.5) with $f=0$. (Recall that (1.6) implies the exponential dichotomy of the semigroup generated by $A$, but that $\sigma(A(t)) \cap i \mathbb{R}=\emptyset$ for $t \geq 0$ does not imply the exponential dichotomy of $U(\cdot, \cdot)$.) The approach of [6] in fact leads to robustness results for the exponential dichotomy of (1.5) under small perturbations defined on $D(A(t))$; see [33, Thm. 4.1] and also [22, Thm. 3.5] for an earlier result in a more regular setting.

Moreover, if $f(t) \rightarrow f_{\infty}$, then the mild solution $u$ of (1.5) converges to the stationary solution at infinity, i.e.,

$$
\lim _{t \rightarrow \infty} u(t)=-A^{-1} f_{\infty}=: u_{\infty}
$$

provided a compatibility condition for $f$ and $x$ holds, which is necessary for the boundedness of $u$. If $f$ is, e.g., Hölder continuous, then the mild solution is a classical one,

$$
\lim _{t \rightarrow \infty} \dot{u}(t)=0, \quad \text { and } \quad \lim _{t \rightarrow \infty} A(t) u(t)=A u_{\infty}
$$

due to [32, Thm. 4.1]. These results extend a theorem by H. Tanabe [35. Thm. 5.6.1]; see also [16] for closely related facts and [32] for further references.

If one wants to extend these theorems to the retarded problem (1.1), several new phenomena appear. First of all, one has to consider the limit problem

$$
\dot{u}(t)=A u(t)+L u_{t}, \quad t \geq 0, \quad u_{0}=\phi \in E .
$$

Recall that the solution of (1.8) is given by $u(t)=[S(t) \phi](0)$, where the semigroup $S(\cdot)$ on $E$ is generated by

$$
B:=\frac{d}{d \xi}, \quad D(B):=\left\{\phi \in C^{1}([-r, 0], X): \phi(0) \in D(A), \phi^{\prime}(0)=A \phi(0)+L \phi\right\} .
$$

It is known that $S(\cdot)$ has an exponential dichotomy if and only if

$$
\sigma(B) \cap i \mathbb{R}=\emptyset \Longleftrightarrow \lambda \in \rho\left(A+L_{\lambda}\right) \quad \forall \lambda \in i \mathbb{R},
$$

where $L_{\lambda} \in \mathcal{L}(X)$ is given by $L_{\lambda} x:=L e_{\lambda} x$ for $\lambda \in \mathbb{C}$ and $e_{\lambda}(\xi):=e^{\lambda \xi},-r \leq \xi \leq 0$. The right hand side of (1.10) replaces the spectral condition (1.6) and can be regarded as a 'generalized characteristic equation' for (1.8). The proofs of these facts are contained in [11, $\S$ VI.6]; see also [36, $\S \S 3.1,3.2]$ and the references therein.

Second, the presence of the retardations $L(t)$ delays the smoothing effect of the parabolic part of (1.1). Moreover, in order to treat the example (1.4) we have to involve the Hölder space in the convergence property (1.3). These points lead to somewhat tedious computations involving deeper regularity properties of the undelayed parabolic equation (1.5) established in [1, 2]. In order to keep the technical difficulties within reasonable bounds, we do not consider delay terms defined on intermediate spaces between $D(A(t))$ and $X$ (which would probably be 
possible). . In addition, the treatment of the inhomogeneous problem requires new 'variation of parameter formulas'; see Section 4.

The main problem, however, is of a more principal nature. The approach of [6] and [32] relies on a perturbation result for exponential dichotomies of nonautonomous evolution equations without delays; see e.g. [7], 32]. Such results are typically based on characterizations of exponential dichotomy, where we prefer a characterization in terms of the spectra of an associated 'evolution semigroup' on the space $C_{0}(\mathbb{R}, X)$; see (2.13) and [7]. Based on (2.13), we recently characterized [15] the exponential dichotomy of a large class of retarded problems by the generalized characteristic equation (2.15). This equivalence (combined with further semigroup theory) then allows us to establish our crucial robustness results for (1.1), Propositions 3.2 and 3.3. Observe that the exponential dichotomy need not persist under small delays if the problem is not parabolic; see [4], 9], [19].

In Theorem 3.6 we then establish our main result: The homogeneous problem (1.1) with $f=0$ has an exponential dichotomy (on a time interval $[a, \infty) \subseteq \mathbb{R}_{+}$) if condition (1.10) holds. In different settings this subject is also treated in [4], 10, [15], [17, [19], [21], 30]. We further refer to [8], [18 §9.5], [21], 24] for related investigations if $X=\mathbb{C}^{n}$. Based on this theorem, it is proved in Section 4 that the mild solution $u$ of the inhomogeneous problem (1.1) converges to the stationary solution at infinity, i.e.,

$$
\lim _{t \rightarrow \infty} u(t)=-\left(A+L_{0}\right)^{-1} f_{\infty} .
$$

To that purpose we have to establish new representation formulas for the mild solution of the inhomogeneous equation, which are inspired by the evolution semigroup approach and by the papers [13, 14, 15, 26]. Finally, if $f$ and $L(\cdot)$ are, e.g., Hölder continuous in time, then the mild solution $u$ of (1.1) is a classical one and satisfies again (1.7) as we show in Section [5] There we also discuss a retarded parabolic partial differential equation.

In the next section we present auxiliary results concerning the solvability and regularity of (1.5) and (1.1) and concerning exponential dichotomy and its characterizations. Unexplained notation can be found in [11. By $c$ we denote a generic constant.

\section{Prerequisites}

Parabolic evolution equations. Let $X$ be a Banach space and $J$ be either $\mathbb{R}$ or $[a, \infty)$. A collection $\{U(t, s): t \geq s, t, s \in J\} \subseteq \mathcal{L}(X)$ is said to be an evolution family if

(a) $U(t, s)=U(t, \tau) U(\tau, s), \quad U(s, s)=I$, and

(b) $(t, s) \mapsto U(t, s)$ is strongly continuous

for $t \geq \tau \geq s$ and $t, \tau, s \in J$. A closed linear operator $A$ on $X$ is called sectorial of type $(\phi, K)$ if it is densely defined and

$$
\|R(\lambda, A)\| \leq \frac{K}{1+|\lambda|} \quad \text { for } \quad \lambda \in \Sigma_{\phi} \subseteq \rho(A)
$$

\footnotetext{
${ }^{*}$ A retardation such as $L(t) \phi=A(t) \phi(-\tau(t))$ would lead to more severe difficulties; e.g., (1.10) does not imply the exponential dichotomy of the corresponding autonomous problem in certain cases; see 5 .
} 
and constants $K \geq 0, \phi \in(\pi / 2, \pi)$, where $\Sigma_{\phi}:=\{0\} \cup\{\lambda \in \mathbb{C} \backslash\{0\}:|\arg \lambda| \leq \phi\}$. Recall that then $A$ generates an exponentially stable analytic semigroup $\left(e^{t A}\right)_{t \geq 0}$. Given $\theta \in(0,1)$ and an operator $A$ such that $A_{w}:=A-w$ is sectorial for some $w \geq 0$, we set

$$
\|x\|_{\theta}^{A}:=\sup _{\lambda \in \Sigma_{\phi}}\left\|\lambda^{\theta} A_{w} R\left(\lambda, A_{w}\right) x\right\| \quad \text { and } \quad X_{\theta}^{A}:=\left\{x \in X:\|x\|_{\theta}^{A}<\infty\right\} .
$$

Note that $\left(X_{\theta}^{A},\|\cdot\|_{\theta}^{A}\right)$ is a Banach space continuously embedded in $X$. Further, $X_{1}^{A}:=D(A)$ is equipped with the graph norm of $A$, and $X_{0}^{A}:=X$. We refer to [3, Chaps. I, V], [11, §II.5], or [23] Chaps. 1, 2] for the interpolation theory of (analytic) semigroups. The following hypothesis was introduced by P. Acquistapace and B. Terreni in [2].

(P) The operators $A_{w}(t)=A(t)-w, t \in J$, are sectorial of the same type $(\phi, K)$ and

$$
\left\|A_{w}(t)^{-1}-A_{w}(s)^{-1}\right\|_{\mathcal{L}\left(X, X_{\nu}^{t}\right)} \leq L|t-s|^{\mu}
$$

for $t, s \in J$ and constants $w, K, L \geq 0, \phi \in\left(\frac{\pi}{2}, \pi\right)$, and $\mu, \nu \in(0,1]$ with $\mu+\nu>1$.

Here we let $X_{\theta}^{t}:=X_{\theta}^{A(t)}$ and $\|x\|_{\theta}^{t}:=\|x\|_{\theta}^{A(t)}$. Under these assumptions it is shown in [1, Thm. 2.3] that there exists an evolution family $U(t, s), t \geq s, t, s \in J$, such that $U(\cdot, s) \in C^{1}((s, \infty), \mathcal{L}(X))$ and $\partial_{t} U(t, s)=A(t) U(t, s)$ for $t>s$. Moreover, $u=U(\cdot, s) x \in C^{1}([s, \infty), X)$ is the unique solution of (1.5) for $x \in D(A(s))$ and $f=0$; see also [2], 3], 37, 38. We say that $A(\cdot)$ generates $U(\cdot, \cdot)$. This evolution family also possesses parabolic regularity in the sense that

$$
\begin{aligned}
\|U(t, s)\|_{\mathcal{L}\left(X_{\theta}^{s}, X_{\theta}^{t}\right)}+(t-s)^{\theta}\|U(t, s)\|_{\mathcal{L}\left(X, X_{\theta}^{t}\right)} & \leq C, \\
\|A(t) U(t, s)\|_{\mathcal{L}\left(X_{\theta}^{s}, X\right)} & \leq C(t-s)^{\theta-1}, \\
\|U(t, s)-I\|_{\mathcal{L}\left(X_{\theta}^{s}, X\right)} & \leq C(t-s)^{\theta}
\end{aligned}
$$

for $0<t-s \leq 1,0 \leq \theta \leq 1$, and a constant $C$ depending only on the constants in $(\mathrm{P})$. In [38, Thms. 2.1, 2.3] similar estimates involving fractional powers are established. In the present context, (2.1) follows by interpolating the cases $\theta=0,1$ in 11, Thm. 2.3], whereas (2.2) and (2.3) are consequences of the representation formula

$$
U(t, s)=e^{(t-s) A(s)}+\int_{s}^{t} Z(\tau, s) d \tau
$$

given in [1, (2.6)], the regularity of analytic semigroups (see [23, §2.2]), and the estimates for $Z(t, s)$ stated in [1, Lemma 2.2]. (To verify (2.2), use that (2.4) yields

$$
A(t) U(t, s)=\partial_{t} U(t, s)=A(s) e^{(t-s) A(s)}+Z(t, s)
$$

for $t>s$.) Similarly one sees that

$$
\left\|A(t) U(t, s)-A\left(t^{\prime}\right) U\left(t^{\prime}, s\right)\right\| \leq C_{\theta} \varepsilon^{-1-\theta}\left(t^{\prime}-t\right)^{\theta}
$$

for $s+\varepsilon \leq t \leq t^{\prime} \leq s+1$, and $\theta \in[0, \mu+\nu-1)$. Note that (2.1) with $\theta=0$ implies the exponential boundedness of $U(t, s)$, i.e., there are constants $M \geq 1$ and $\omega \in \mathbb{R}$ such that

$$
\|U(t, s)\| \leq M e^{\omega(t-s)} \quad \text { for } t \geq s
$$


For $f \in L_{l o c}^{1}(J, X)$ and $x \in X$ the mild solution of (1.5) is defined by

$$
u(t)=U(t, s) x+\int_{s}^{t} U(t, \tau) f(\tau) d \tau .
$$

Every classical, and even strong, solution of (1.5) is a mild one by [1, Prop. 3.2]. Conversely, the mild solution is continuously differentiable, $u(t) \in D(A(t))$, and it satisfies (1.5) for $t \geq s$ if $x \in D(A(s))$ and $f$ is Hölder continuous or bounded with respect to interpolation norms due to [2, Thms. 6.1,6.2].

Retarded evolution equations. We now turn our attention to the retarded problem (1.1). Let $U(\cdot, \cdot)$ be an exponentially bounded evolution family on $X$ with $J=[a, \infty), \mathbb{R}$ and let $L(\cdot) \in C_{b}\left(J, \mathcal{L}_{s}(E, X)\right)$. Here and below we use the notation introduced in Section 1. Define the evolution family $\tilde{U}(\cdot, \cdot)$ on $E$ by

$$
(\tilde{U}(t, s) \phi)(\xi):= \begin{cases}\phi(t+\xi-s), & -r \leq \xi \leq s-t, \\ U(t+\xi, s) \phi(0), & s-t \leq \xi \leq 0\end{cases}
$$

for $\xi \in[-r, 0], \phi \in E$, and $t \geq s, s \in J$. Then there exists a unique exponentially bounded evolution family $V(\cdot, \cdot)$ on $E$ satisfying

$$
(V(t, s) \phi)(\xi)=(\tilde{U}(t, s) \phi)(\xi)+\int_{s}^{(t+\xi) \vee s} U(t+\xi, \tau) L(\tau) V(\tau, s) \phi d \tau
$$

for $\xi \in[-r, 0], \phi \in E, t \geq s$, and $s \in J$, where $a \vee b:=\max \{a, b\}$. This result is more or less known and proved in e.g. [30, Prop. 3.2]. In the autonomous case, where $L(t)=L$ and $U(t, s)=e^{(t-s) A}$, we obtain $V(t, s)=S(t-s)$ for the semigroup $S(\cdot)$ generated by the operator $B$ defined in (1.9); see Lemmas VI.6.2 and VI.6.5 in [1] or [36, Thm. 3.1.5]. Gronwall's inequality and the estimate (2.6) with $\omega \geq 0$ imply that

$$
\|V(t, s)\| \leq M \exp \left[\left(\omega+M\|L(\cdot)\|_{\infty}\right)(t-s)\right] .
$$

Given $\phi \in E$ and $s \in J$, we set

$$
u(t):= \begin{cases}\phi(t-s), & t \in[s-r, s], \\ V(t, s) \phi(0), & t \geq s .\end{cases}
$$

Formulas (2.9) and (2.8) yield $u_{t}=V(t, s) \phi$ for $t \geq s$, and $u$ is thus the unique continuous solution of

$$
u(t)=U(t, s) \phi(0)+\int_{s}^{t} U(t, \tau) L(\tau) u_{\tau} d \tau
$$

for $t \geq s$. Further, let $f \in L_{l o c}^{1}(J, X)$. In analogy to (2.7), we call a function $u \in C([s-r, \infty), X)$ satisfying

$$
\begin{aligned}
& u(t)=U(t, s) \phi(0)+\int_{s}^{t} U(t, \tau)\left(L(\tau) u_{\tau}+f(\tau)\right) d \tau, \quad t \geq s, \\
& u(t)=\phi(t-s), \quad s-r \leq t \leq s
\end{aligned}
$$

the mild solution of the inhomogeneous problem (2.11) (or of (1.1) if $U(\cdot, \cdot)$ is generated by $A(\cdot))$. By a standard fix point argument one easily constructs a mild solution which is unique by Gronwall's inequality; see e.g. [12, Thm. 3.2]. In Section 4 we derive representation formulas for the mild solutions, whereas in 
Proposition 5.2 its regularity is investigated in the parabolic case. For more results in this context we refer to [10, [13], [14, [15], 25], [26], 27], 34], [36, Chap. 4].

Exponential dichotomy. An evolution family $U(\cdot, \cdot)$ on $X$ with $J=[a, \infty)$ or $\mathbb{R}$ is said to have an exponential dichotomy (or to be hyperbolic) if there are projections $P(\cdot) \in C_{b}\left(J, \mathcal{L}_{s}(X)\right)$ and constants $N, \delta>0$ such that

(a) $U(t, s) P(s)=P(t) U(t, s)$,

(b) the restriction $U_{Q}(t, s): Q(s) X \rightarrow Q(t) X$ of $U(t, s)$ is invertible (and we set $\left.U_{Q}(s, t):=U_{Q}(t, s)^{-1}\right)$,

(c) $\|U(t, s) P(s)\| \leq N e^{-\delta(t-s)}$ and $\left\|U_{Q}(s, t) Q(t)\right\| \leq N e^{-\delta(t-s)}$,

for $t \geq s$ and $s \in J$. Here and below we let $Q=I-P$ for a projection $P$. In the case that $P(t)=I$ for $t \in J$ we call $U(\cdot, \cdot)$ exponentially stable. We also need Green's function

$$
\Gamma(t, s):= \begin{cases}U(t, s) P(s), & t \geq s, t, s \in J \\ -U_{Q}(t, s) Q(s), & t<s, t, s \in J\end{cases}
$$

corresponding to a hyperbolic evolution family $U(\cdot, \cdot)$ with projections $P(\cdot)$.

The dichotomy projections are uniquely determined by (a)-(c) if $J=\mathbb{R},[29$ Cor. 3.3]. This uniqueness fails for $J=[a, \infty)$. If $U(t, s)=T(t-s)$ for $t \geq s$, $J=\mathbb{R}$, and a $C_{0}$-semigroup $T(\cdot)$, then $P(t)=P$ does not depend on $t$ by [29] Cor. 3.3] so that the standard spectral theory for semigroups applies (compare [11] $\S[\mathrm{IV} .3, \mathrm{~V} .1])$.

Let $U(\cdot, \cdot)$ be an exponentially bounded evolution family on $X$ with $J=\mathbb{R}$. As can be seen by simple ODE examples, see e.g. [11 Ex. VI.9.9], we cannot hope to characterize the exponential dichotomy of an evolution family by the spectra of the operators generating it. Instead we use the spectra of the associated evolution semigroup given by

$$
\left(T_{U}(t) f\right)(s):=U(s, s-t) f(s-t), \quad t \geq 0, s \in \mathbb{R}, f \in C_{0}(\mathbb{R}, X),
$$

and its generator $G_{U}$; cf. [7] or [11, §VI.9]. It is not difficult to show that $T_{U}(\cdot)$ is in fact a $C_{0}$-semigroup on $C_{0}(\mathbb{R}, X)$ and that

$$
\left(R\left(\lambda, G_{U}\right) f\right)(t)=\int_{-\infty}^{t} U_{\lambda}(t, s) f(s) d s
$$

for $t \in \mathbb{R}, f \in C_{0}(\mathbb{R}, X)$, and $\operatorname{Re} \lambda>\omega$, where $\omega$ is given as in (2.6) and $U_{\lambda}(t, s):=$ $e^{-\lambda(t-s)} U(t, s)$. A result by R. Rau, Y. Latushkin, and S. Montgomery-Smith says that

$$
U(\cdot, \cdot) \text { is hyperbolic } \Longleftrightarrow 1 \in \rho\left(T_{U}\left(t_{0}\right)\right) \text { for some } t_{0}>0 \Longleftrightarrow 0 \in \rho\left(G_{U}\right)
$$

and that then the dichotomy projections are given by

$$
P(\cdot)=\frac{1}{2 \pi i} \int_{\mathbb{T}} R\left(\lambda, T_{U}\left(t_{0}\right)\right) d \lambda,
$$

for the unit circle $\mathbb{T}$; see [7, Thm. 3.17] or [11, Thm. VI.9.18]. Assume further that $L(\cdot) \in C_{b}\left(\mathbb{R}, \mathcal{L}_{s}(E, X)\right)$ and let $V(\cdot, \cdot)$ solve (2.9). Evaluating the equivalence (2.13) for the evolution semigroup on $C_{0}(\mathbb{R}, E)$ induced by $V(t, s)$, it was proved in [15, Cor. 3.6] that

(2.15) $\quad V(\cdot, \cdot)$ is hyperbolic on $E \Longleftrightarrow G_{U}+\widehat{L(\cdot)}$ is invertible on $C_{0}(\mathbb{R}, X)$, where $(\widehat{L(\cdot)} f)(t):=L(t) f_{t}$ for $f \in C_{0}(\mathbb{R}, X)$ and $D\left(G_{U}+\widehat{L(\cdot)}\right):=D\left(G_{U}\right)$. 
Finally we state a result needed in Sections 4 and 5 .

Proposition 2.1. Let $U(\cdot, \cdot)$ be an exponentially bounded evolution family on a Banach space $X$ with $J=\mathbb{R}$ and let $A$ generate the $C_{0}$-semigroup $S(\cdot)$. Assume that $U(\cdot, \cdot)$ and $S(\cdot)$ have exponential dichotomy with projections $P(t)$ and $P$, respectively, and that $U(s+t, s) \rightarrow S(t)$ strongly for $t \geq 0$ as $s \rightarrow \infty$. Then $P(s) \rightarrow P$ strongly as $s \rightarrow \infty$.

Proof. Let $\tilde{F}:=\left\{f \in C(\mathbb{R}, X): f(t) \rightarrow 0\right.$ as $t \rightarrow-\infty, f(t) \rightarrow f_{\infty}$ as $\left.t \rightarrow \infty\right\}$ be endowed with the sup-norm. Clearly,

$$
\left(\tilde{T}_{U}(t) f\right)(s):=U(s, s-t) f(s-t), \quad t \geq 0, s \in \mathbb{R}, f \in \tilde{F},
$$

defines an exponentially bounded semigroup $\tilde{T}_{U}(\cdot)$ on $\tilde{F}$. Its restriction to $F:=$ $C_{0}(\mathbb{R}, X)$ is the evolution semigroup $T_{U}(\cdot)$. The spaces $\tilde{F}$ and $F \times X=: \hat{F}$ are isomorphic via

$$
\Phi: \tilde{F} \rightarrow \hat{F}, f \mapsto\left(f-M f_{\infty}, f_{\infty}\right),
$$

where $M x:=\varphi(\cdot) x$ for a fixed function $\varphi \in C(\mathbb{R})$ with support in $\mathbb{R}_{+}$and $\lim _{t \rightarrow \infty} \varphi(t)=1$. We consider the induced semigroup $\hat{T}_{U}(\cdot)$ on $\hat{F}$ given by

$$
\hat{T}_{U}(t):=\Phi \tilde{T}_{U}(t) \Phi^{-1}=\left(\begin{array}{cc}
T_{U}(t) & \tilde{T}_{U}(t) M-M S(t) \\
0 & S(t)
\end{array}\right), \quad t \geq 0 .
$$

By the assumption and (2.13), $\lambda-T_{U}(1)$ and $\lambda-S(1)$ are invertible for $|\lambda|=1$; hence $\lambda \in \rho\left(\hat{T}_{U}(1)\right)$. This means that $\hat{T}_{U}(\cdot)$ and $\tilde{T}_{U}(\cdot)$ are hyperbolic with spectral projections $\hat{\mathcal{P}}$ and $\tilde{\mathcal{P}}$, respectively. On the other hand, formula (2.16) defines a semigroup $T_{U}^{b}(\cdot)$ on $C_{b}(\mathbb{R}, X)$ which is hyperbolic with projection

$$
P(\cdot)=\mathcal{P}^{b}=\frac{1}{2 \pi i} \int_{\mathbb{T}} R\left(\lambda, T_{U}^{b}(1)\right) d \lambda
$$

by [28, Thm. 5.6] and the exponential dichotomy of $U(\cdot, \cdot)$. Since $R\left(\lambda, T_{U}^{b}(1)\right) f=$ $R\left(\lambda, \tilde{T}_{U}(1)\right) f$ for $f \in \tilde{F}$, we obtain $\tilde{\mathcal{P}}=\mathcal{P}^{b}=P(\cdot)$. Therefore $P(t)$ converges strongly to a projection $P^{\prime}$ as $t \rightarrow \infty$. Finally,

$$
\begin{aligned}
\hat{\mathcal{P}} & =\frac{1}{2 \pi i} \int_{\mathbb{T}} R\left(\lambda, \hat{T}_{U}(1)\right) d \lambda=\left(\begin{array}{cc}
P(\cdot) & * \\
0 & P
\end{array}\right) \\
& =\frac{1}{2 \pi i} \int_{\mathbb{T}} \Phi R\left(\lambda, \tilde{T}_{U}(1)\right) \Phi^{-1} d \lambda=\Phi P(\cdot) \Phi^{-1}=\left(\begin{array}{cc}
P(\cdot) & * \\
0 & P^{\prime}
\end{array}\right)
\end{aligned}
$$

so that $P=P^{\prime}$.

\section{Exponential DICHOTOMY OF RETARDED EVOLUTION EQUATIONS}

We start with two robustness results for exponential dichotomy, where first $L(\cdot)$ and then $U(\cdot, \cdot)$ is varied employing different techniques. Proposition 3.2 shows in particular that dichotomy is not sensitive to small delays in the parabolic case; see [4, Thm. 4.2] and [19] for related autonomous results. We point out that in general, exponential stability can be destroyed by arbitrarily small delays; see [4], [9], [19]. 
The next preliminary lemma allows us to prove the uniformity of the dichotomy constants needed in Proposition 3.8.

Lemma 3.1. Let $U(\cdot, \cdot)$ be an evolution family with $J=\mathbb{R}$ satisfying (2.6) with $\omega \geq 0$ and $L(\cdot) \in C_{b}\left(\mathbb{R}, \mathcal{L}_{s}(E, X)\right)$. Assume that the evolution family $V(\cdot, \cdot)$ solving (2.9) on $E$ has an exponential dichotomy with constants $N, \delta>0$. Then $N \leq N_{0}$ and $\delta \geq \delta_{0}>0$ where $N_{0}$ and $\delta_{0}$ only depend on $M, \omega,\|L(\cdot)\|_{\infty},\left\|\left(G_{U}+\widehat{L(\cdot)}\right)^{-1}\right\|$. Conversely,

$$
\left\|\left(G_{U}+\widehat{L(\cdot)}\right)^{-1}\right\| \leq 4 N / \delta
$$

Proof. Due to [31, Lemma 3.1], $N$ and $\delta$ only depend on the exponential estimate of $V(\cdot, \cdot)$ and the norm of $G_{V}^{-1}$. The first assertion thus follows from (2.10) and the formula

$$
\begin{aligned}
& \left(G_{V}^{-1} f\right)(t)=\left[\left(G_{U}+\widehat{L(\cdot)}\right)^{-1}(f(\cdot, 0)+L(\cdot) R f)\right]_{t}-(R f)(t), \\
& (R f)(t, \xi)=\int_{\xi}^{0} f(\xi-\tau+t, \tau) d \tau, \quad f \in C_{0}(\mathbb{R}, E), t \in \mathbb{R}, \xi \in[-r, 0],
\end{aligned}
$$

shown in [15, Thm.3.5]. The proof of this theorem also yields that

$$
\left(G_{U}+\widehat{L(\cdot)}\right)^{-1} \varphi=\left(G_{V}^{-1} \Phi_{L} \varphi\right)(\cdot, 0)
$$

for $\varphi \in C_{0}(\mathbb{R}, X)$ and a map $\Phi_{L}: C_{0}(\mathbb{R}, X) \rightarrow C_{0}(\mathbb{R}, E)$ with norm less than 2; cf. [15, (3.12)]. This implies the second assertion since $\left\|G_{V}^{-1}\right\| \leq 2 N / \delta$ by [11, Thm. VI.9.18].

Proposition 3.2. Let $A(t), t \in \mathbb{R}$, satisfy $(P)$ and generate $U(\cdot, \cdot)$ on $X$. Let $V_{k}(\cdot, \cdot)$ solve (2.9) on $E$ for $U(\cdot, \cdot)$ and $L_{k}(\cdot) \in C_{b}\left(\mathbb{R}, \mathcal{L}_{s}(E, X)\right), k=1,2$. Assume that

$$
q:=\sup _{t \in \mathbb{R}}\left\|L_{1}(t)-L_{2}(t)\right\|_{\mathcal{L}\left(C^{\beta}([-r, 0], X), X\right)}<\infty
$$

for some $\beta \in[0,1)$ and that $V_{1}(\cdot, \cdot)$ has an exponential dichotomy. Then there is a number $q_{0}>0$ such that $V_{2}(\cdot, \cdot)$ also has an exponential dichotomy with constants $N, \delta>0$ provided that $q \leq q_{0}$. Moreover, $N \leq N_{0}$ and $\delta \geq \delta_{0}>0$ where $N_{0}$ and $\delta_{0}$ only depend on $U(\cdot, \cdot), L_{1}(\cdot),\left\|L_{2}(\cdot)\right\|_{\infty}$, and $q_{0}$.

Proof. In view of (2.15) we have to show that $G_{U}+\widehat{L_{2}(\cdot)}$ with domain $D\left(G_{U}\right)$ is invertible on $C_{0}(\mathbb{R}, X)$ knowing that $G_{U}+\widehat{L_{1}(\cdot)}$ is invertible. Fix $\lambda>\omega$, where $\omega \geq 0$ is given by (2.6) . Then $\lambda \in \rho\left(G_{U}\right),\left(\lambda-G_{U}\right)\left(G_{U}+\widehat{L_{1}(\cdot)}\right)^{-1}$ is bounded, and

$$
\begin{aligned}
G_{U} & +\widehat{L_{2}(\cdot)} \\
& =\left[I-\left(\widehat{L_{1}(\cdot)}-\widehat{L_{2}(\cdot)}\right) R\left(\lambda, G_{U}\right)\left(\lambda-G_{U}\right)\left(G_{U}+\widehat{L_{1}(\cdot)}\right)^{-1}\right]\left(G_{U}+\widehat{L_{1}(\cdot)}\right) .
\end{aligned}
$$


We deduce from (3.1), (2.12), (2.3), and (2.1) that

$$
\begin{aligned}
\left\|\left(\widehat{L_{1}(\cdot)}-\widehat{L_{2}(\cdot)}\right) R\left(\lambda, G_{U}\right) f\right\|_{\infty} \\
\leq q\left[\left\|R\left(\lambda, G_{U}\right) f\right\|_{\infty}\right. \\
\left.\quad+\sup _{\substack{-r \leq \eta \leq \xi \leq 0 \\
t \in \mathbb{R}}}|\xi-\eta|^{-\beta}\left\|R\left(\lambda, G_{U}\right) f(t+\xi)-R\left(\lambda, G_{U}\right) f(t+\eta)\right\|\right] \\
\leq q\left[c\|f\|_{\infty}+\sup _{\substack{-r \leq \eta \leq \xi \leq 0 \\
t \in \mathbb{R}}}|\xi-\eta|^{-\beta}\left(\int_{t+\eta}^{t+\xi}\left\|U_{\lambda}(t+\xi, \tau) f(\tau)\right\| d \tau\right.\right. \\
\left.\left.\quad+\int_{-\infty}^{t+\eta}\left\|\left(U_{\lambda}(t+\xi, t+\eta)-I\right) U_{\lambda}(t+\eta, \tau) f(\tau)\right\| d \tau\right)\right] \\
\leq c q\|f\|_{\infty}
\end{aligned}
$$

for $f \in C_{0}(\mathbb{R}, X)$ and constants $c>0$ independent of $q$ and $f$. Thus (3.2) implies the invertibility of $G_{U}+\widehat{L_{2}(\cdot)}$ for sufficiently small $q$. The final assertion is an easy consequence of the previous lemma and (3.2).

Proposition 3.3. Let $U(\cdot, \cdot)$ and $U_{n}(\cdot, \cdot), n \in \mathbb{N}$, be evolution families on $X$ with $J=\mathbb{R}$ satisfying (2.6) uniformly in $n$, and let $L_{n}(\cdot) \in C_{b}\left(\mathbb{R}, \mathcal{L}_{s}(E, X)\right)$ be uniformly bounded in $n$. Let $V_{n}(\cdot, \cdot)$, respectively $\tilde{V}_{n}(\cdot, \cdot)$, solve (2.9) on $E$ with $J=\mathbb{R}$ for $L_{n}(\cdot)$ and $U_{n}(\cdot, \cdot)$, respectively $U(\cdot, \cdot)$. Assume that

$$
\lim _{n \rightarrow \infty} \sup _{s \in \mathbb{R}}\left\|U_{n}(s+t, s)-U(s+t, s)\right\|_{\mathcal{L}(X)}=0
$$

for all $t \geq 0$ and that $\tilde{V}_{n}(\cdot, \cdot)$ is hyperbolic with uniform constants. Then $V_{n}(\cdot, \cdot)$ is hyperbolic for large $n$ and the dichotomy constants can be chosen independent of $n$.

Proof. Let $G_{U_{n}}=G_{n}$ be the generator of the evolution semigroup $T_{n}(\cdot)$ on $C_{0}(\mathbb{R}, X)$ corresponding to $U_{n}(\cdot, \cdot)$. Due to $(2.15)$ we know that $\tilde{H}_{n}:=G_{U}+\widehat{L_{n}(\cdot)}$ with domain $D\left(G_{U}\right)$ is invertible and we have to show the invertibility of $H_{n}:=G_{n}+\widehat{L_{n}(\cdot)}$ with domain $D\left(G_{n}\right)$ for large $n$. Let $R_{n}(\cdot)$ and $\tilde{R}_{n}(\cdot)$ be the semigroups on $C_{0}(\mathbb{R}, X)$ generated by $H_{n}$ and $\tilde{H}_{n}$, respectively. By standard perturbation theory, both semigroups are exponentially bounded uniformly in $n$ (with a common exponential bound $\tilde{\omega}$ ) and we have

$$
\begin{aligned}
R_{n}(t)-\tilde{R}_{n}(t)= & T_{n}(t)-T_{U}(t)+\int_{0}^{t}\left(T_{n}(t-s)-T_{U}(t-s)\right) \widehat{L_{n}(\cdot)} R_{n}(s) d s \\
& +\int_{0}^{t} T_{U}(t-s) \widehat{L_{n}(\cdot)}\left(R_{n}(s)-\tilde{R}_{n}(s)\right) d s
\end{aligned}
$$

for $t \geq 0$. Observe that $t \mapsto\left\|R_{n}(t)-\tilde{R}_{n}(t)\right\|$ is lower semicontinuous and thus measurable. Therefore the function

$$
\varphi: \mathbb{R}_{+} \rightarrow \mathbb{R}_{+} ; \quad \varphi(t)=\varlimsup_{n \rightarrow \infty}\left\|R_{n}(t)-\tilde{R}_{n}(t)\right\|
$$

is measurable and also locally bounded. Assumption (3.3) implies that $T_{n}(t) \rightarrow$ $T_{U}(t)$ in operator norm for $t \geq 0$ as $n \rightarrow \infty$. We thus deduce from (3.4)

$$
\varphi(t) \leq c \int_{0}^{t} \varphi(s) d s \quad \text { for } t \geq 0
$$


using Fatou's lemma. Therefore $R_{n}(t)-\tilde{R}_{n}(t)$ tends to 0 in operator norm for $t \geq 0$ due to Gronwall's inequality. This yields $R\left(\lambda, H_{n}\right)-R\left(\lambda, \tilde{H}_{n}\right) \rightarrow 0$ in $\mathcal{L}\left(C_{0}(\mathbb{R}, X)\right)$ as $n \rightarrow \infty$ for a fixed $\lambda>\tilde{\omega}$. Observe that

$$
\begin{gathered}
I-\lambda R\left(\lambda, H_{n}\right)=\left\{I-\lambda\left(R\left(\lambda, H_{n}\right)-R\left(\lambda, \tilde{H}_{n}\right)\right)\left[I-\lambda R\left(\lambda, \tilde{H}_{n}\right)\right]^{-1}\right\}\left[I-\lambda R\left(\lambda, \tilde{H}_{n}\right)\right], \\
{\left[I-\lambda R\left(\lambda, \tilde{H}_{n}\right)\right]^{-1}=I-\lambda \tilde{H}_{n}^{-1} .}
\end{gathered}
$$

As a result, $I-\lambda R\left(\lambda, H_{n}\right)$ has a uniformly bounded inverse for large $n$ due to Lemma 3.1 and the assumptions. Hence

$$
H_{n}^{-1}=R\left(\lambda, H_{n}\right)\left[\lambda R\left(\lambda, H_{n}\right)-I\right]^{-1}
$$

exists and is uniformly bounded for large $n$. The assertion now follows from (2.15) and Lemma 3.1.

We return to the asymptotically autonomous case making the following assumptions.

(H1) The operators $A(t), t \geq 0$, on $X$ satisfy (P) and there is an operator $A$ on $X$ such that $A-w$ is sectorial and

$$
o_{A}(t):=\sup _{s \geq t}\|R(w, A(s))-R(w, A)\|_{\mathcal{L}(X)} \longrightarrow 0 \quad \text { as } t \rightarrow \infty .
$$

(H2) There are operators $L(\cdot) \in C_{b}\left(\mathbb{R}_{+}, \mathcal{L}_{s}(E, X)\right)$ and $L \in \mathcal{L}(E, X)$ such that

$$
o_{L}(t):=\sup _{s \geq t}\|L(s)-L\|_{\mathcal{L}\left(C^{\beta}([-r, 0], X), X\right)} \longrightarrow 0
$$

as $t \rightarrow \infty$ for some $\beta \in[0,1)$.

In the remainder of this section $U(\cdot, \cdot)$ is the evolution family on $X$ generated by $A(\cdot), T(\cdot)$ is the semigroup on $X$ generated by $A, V(\cdot, \cdot)$ is the evolution family on $E$ solving (2.9), and $S(\cdot)$ is the semigroup on $E$ generated by $B$ (see (1.9)).

In order to establish our results on exponential dichotomy, we need some convergence and regularity properties of $U(\cdot, \cdot)$ and $V(\cdot, \cdot)$, respectively. For $a \geq 0$ we introduce the evolution family

$$
U_{a}(t, s):= \begin{cases}U(t, s), & t \geq s \geq a, \\ U(t, a) T(a-s), & t>a>s, \\ T(t-s), & a \geq t \geq s,\end{cases}
$$

on $X$. We further define

$$
F_{\theta, h}^{t}:=\left\{\phi \in C^{h}([-r, 0], X): \phi(0) \in X_{\theta}^{t}\right\}
$$

and

$$
F_{\theta, h}^{A}:=\left\{\phi \in C^{h}([-r, 0], X): \phi(0) \in X_{\theta}^{A}\right\}
$$

for $t \geq 0$ and $h, \theta \in[0,1]$ and endow these spaces with their natural norms.

The first assertion in the next lemma follows from [6] Thm. 4.7], see also [32. Lemma 3.2]. The second assertion is a consequence of the first one, compare the proofs of [6, Prop. 6.1] or [32, Thm. 3.3].

Lemma 3.4. Let (H1) hold. Then $U(s+t, s) \rightarrow T(t)$ in $\mathcal{L}(X)$ as $s \rightarrow \infty$ uniformly for $t \in\left[t_{0}, t_{1}\right] \subseteq(0, \infty)$. Moreover, $U_{a}(s+t, s) \rightarrow T(t)$ in $\mathcal{L}(X)$ as a $\rightarrow \infty$ uniformly for $s \in \mathbb{R}$ and $t \in\left[t_{0}, t_{1}\right] \subseteq(0, \infty)$. 
Lemma 3.5. Let $A(t), t \geq 0$, satisfy $(P), L(\cdot) \in C_{b}\left(\mathbb{R}_{+}, \mathcal{L}_{s}(E, X)\right), 0 \leq h \leq \theta<1$, and $\delta>0$. Then $V(t, s): F_{\theta, h}^{s} \rightarrow F_{\theta, h}^{t}, t \geq s \geq 0$, is exponentially bounded and $V(t, s): E \rightarrow F_{\theta, h}^{t}$ is uniformly bounded for $r+\delta \leq t-s \leq t_{0}$. Moreover, $V(t, s): F_{\theta, h}^{s} \rightarrow F_{0, h}^{t}, t \geq s \geq 0$, is exponentially bounded for $0 \leq h \leq \theta \leq 1$.

Proof. Let $s, t_{0} \geq 0,0 \leq h \leq \theta<1$, and $\delta>0$. From (2.1) and (2.9) we easily deduce

$$
\begin{aligned}
\|[V(t, s) \phi](0)\|_{\theta}^{t} & \leq \begin{cases}c\|\phi(0)\|_{\theta}^{s}+c \int_{s}^{t}(t-\tau)^{-\theta}\|\phi\|_{E} d \tau, & s \leq t \leq s+t_{0}, \\
c\|\phi(0)\|+c \int_{s}^{t}(t-\tau)^{-\theta}\|\phi\|_{E} d \tau, & s+\delta \leq t \leq s+t_{0},\end{cases} \\
& \leq \begin{cases}c\|\phi\|_{F_{\theta, 0}^{s}}, & s \leq t \leq s+t_{0}, \\
c\|\phi\|_{E}, & s+\delta \leq t \leq s+t_{0},\end{cases}
\end{aligned}
$$

where $c$ does not depend on $\phi, s$, and $t$ as indicated above (but possibly on $t_{0}$ and $\delta)$. For $0 \geq \xi \geq \eta \geq-r$ and $t+\eta \geq s$, we can write

$$
\begin{aligned}
& {[V(t, s) \phi](\xi)-[V(t, s) \phi](\eta)} \\
& \quad=(U(t+\xi, t+\eta)-I)[V(t+\eta, s) \phi](0)+\int_{t+\eta}^{t+\xi} U(t+\xi, \tau) L(\tau) V(\tau, s) \phi d \tau .
\end{aligned}
$$

Then (2.3) and (3.6) imply

$$
\|[V(t, s) \phi](\xi)-[V(t, s) \phi](\eta)\| \leq \begin{cases}c|\xi-\eta|^{h}\|\phi\|_{F_{h, 0}^{s}}, & s \leq t \leq s+t_{0}, \\ c|\xi-\eta|^{h}\|\phi\|_{E}, & s+r+\delta \leq t \leq s+t_{0} .\end{cases}
$$

If $t+\xi \geq s \geq t+\eta$, we obtain analogously

$$
\begin{gathered}
{[V(t, s) \phi](\xi)-[V(t, s) \phi](\eta)=U(t+\xi, s) \phi(0)-\phi(0)+\phi(0)-\phi(t+\eta-s)} \\
+\int_{s}^{t+\xi} U(t+\xi, \tau) L(\tau) V(\tau, s) \phi d \tau \\
\|[V(t, s) \phi](\xi)-[V(t, s) \phi](\eta)\| \leq c|\xi-\eta|^{h}\|\phi\|_{F_{h, h}^{s}}
\end{gathered}
$$

for $s \leq t \leq s+t_{0}$ since $0 \leq s-t-\eta, t+\xi-s \leq \xi-\eta$ in this case. The lemma is an immediate consequence of the above estimates and $X_{\theta}^{s} \hookrightarrow X_{h}^{s}$; see e.g. [23] Prop. $1.2 .3]$.

We can now prove that $V(\cdot, \cdot)$ inherits the exponential dichotomy of $S(\cdot)$ which has been characterized in (1.10). For the undelayed problem this result was shown in [6 Thms. 6.2, 6.3] (in a more general situation) and in [32, Thm. 3.3] (under slightly stronger assumptions).

Theorem 3.6. Assume that (H1), (H2), and $\sigma(B) \cap i \mathbb{R}=\emptyset$ hold. Then the evolution family $V(\cdot, \cdot)$ solving (2.9) is hyperbolic on an interval $[a, \infty) \subseteq \mathbb{R}_{+}$with projections $P_{V}(s)$. Moreover, $V(s+t, s) \rightarrow S(t)$ and $P_{V}(s) \rightarrow P_{S}$ strongly on $E$ and uniformly for $t \in\left[0, t_{0}\right]$ as $s \rightarrow \infty$, where $P_{S}$ is the dichotomy projection of $S(\cdot)$.

Proof. (1) Given $a \geq 0$, we define $U_{a}(\cdot, \cdot)$ as in (3.5) and

$$
L_{a}(t):= \begin{cases}L(t), & t \geq a, \\ (t-a+1) L(t)+(a-t) L, & a-1<t<a, \\ L, & t \leq a-1 .\end{cases}
$$


Note that $L_{a}(\cdot) \in C_{b}\left(\mathbb{R}, \mathcal{L}_{s}(E, X)\right)$ and

$$
\left\|L_{a}(t)-L\right\|_{\mathcal{L}\left(C^{\beta}([-r, 0], X), X\right)} \leq \begin{cases}o_{L}(a-1), & t>a-1, \\ 0, & t \leq a-1,\end{cases}
$$

by (H2). We apply Proposition 3.2 with $U(t, s)$ replaced by $T(t-s), L_{1}(t)$ by $L$, and $L_{2}(t)$ by $L_{a}(t)$. Consequently there exists an $a_{0} \geq 0$ such that the evolution family solving (2.9) for $T(t-s)$ and $L_{a}(t)$ has an exponential dichotomy for $a \geq a_{0}$. Lemma 3.4 and Proposition 3.3 (applied to $T(t-s), U_{a}(t, s)$, and $L_{a}(t)$ ) show that there is a number $a_{1} \geq a_{0}$ such that the evolution family $V_{a}(t, s)$ solving (2.9) for $U_{a}(t, s)$ and $L_{a}(t)$ has an exponential dichotomy if $a \geq a_{1}$. This implies the first assertion since $V(t, s)=V_{a}(t, s)$ for $t \geq s \geq a$ by the uniqueness of (2.9).

(2) Let $\phi \in F_{1, \beta}^{s}, s \geq a, t \in\left[0, t_{0}\right]$, and $\xi \in[-r, 0]$ with $t+\xi \geq 0$. Then (2.9) yields

$$
\begin{aligned}
V(s+t, s) & \phi(\xi)-S(t) \phi(\xi) \\
=U( & (s+t+\xi, s) \phi(0)-T(t+\xi) \phi(0) \\
& +\int_{s}^{s+t+\xi}(U(s+t+\xi, \tau)-T(s+t+\xi-\tau)) L(\tau) V(\tau, s) \phi d \tau \\
& +\int_{s}^{s+t+\xi} T(s+t+\xi-\tau)(L(\tau)-L) V(\tau, s) \phi d \tau \\
\quad & +\int_{s}^{s+t+\xi} T(s+t+\xi-\tau) L(V(\tau, s) \phi-S(\tau-s) \phi) d \tau . \\
=: & S_{1}+S_{2}+S_{3}+S_{4} .
\end{aligned}
$$

Observe that

$$
\begin{aligned}
U(s+h, s)-T(h)= & U(s+h, s)-e^{h A(s)} \\
& +\frac{1}{2 \pi i} \int_{\Gamma} e^{\lambda h} A_{w} R(\lambda, A)\left(A_{w}^{-1}-A_{w}(s)^{-1}\right) A_{w}(s) R(\lambda, A(s)) d \lambda
\end{aligned}
$$

for a suitable path $\Gamma$ in $\mathbb{C}$. Formula (2.6) and Lemma 2.2 of [1] thus imply

$$
\|U(s+t+\xi, s) \phi(0)-T(t+\xi) \phi(0)\| \leq c^{\prime}\left((t+\xi)^{\mu+\nu-1}+o_{A}(s)\right)\|\phi(0)\|_{1}^{s}
$$

for a constant independent of $s, \xi, t \in\left[0, t_{0}\right]$, and $\phi$. Let $\delta>0$ and $\tau_{0}:=\left(\frac{\delta}{2 c^{\prime}}\right)^{\frac{1}{\mu+\nu-1}}$. Using the above estimate for $0 \leq t+\xi \leq \tau_{0}$ and Lemma 3.4 for $\tau_{0} \leq t+\xi \leq t_{0}$, we obtain a number $s_{1}(\delta) \geq a$ such that

$$
\left\|S_{1}\right\| \leq \delta\|\phi(0)\|_{1}^{s}
$$

for $s \geq s_{1}(\delta)$. We can further write

$$
S_{2}=\int_{s}^{s \vee(s+t+\xi-\delta)} \cdots d \tau+\int_{s \vee(s+t+\xi-\delta)}^{s+t+\xi} \cdots d \tau
$$


Combining (3.7)-(3.9) with (H2) and Lemmas 3.4 and 3.5, one finds $s_{2}(\delta) \geq s_{1}(\delta)$ such that

$$
\begin{aligned}
& \|V(s+t, s) \phi-S(t) \phi\|_{E} \\
& \leq c \delta\|\phi\|_{F_{1,0}^{s}}+c o_{L}(s) \sup _{0 \leq \tau \leq t}\|V(\tau+s, s) \phi\|_{C^{\beta}} \\
& \quad+c \int_{s}^{s+t}\|V(s+\tau, s) \phi-S(\tau) \phi\|_{E} d \tau \\
& \leq c \delta\|\phi\|_{F_{1, \beta}^{s}}+c \int_{s}^{s+t}\|V(s+\tau, s) \phi-S(\tau) \phi\|_{E} d \tau
\end{aligned}
$$

for $s \geq s_{2}(\delta)$, where the constants $c$ do not depend on $\delta, \phi, s$, and $t \in\left[0, t_{0}\right]$. So Gronwall's equality shows that

$$
\|V(s+t, s) \phi-S(t) \phi\|_{E} \leq c \delta\|\phi\|_{F_{1, \beta}^{s}}
$$

for $s \geq s_{2}(\delta)$. Given an $\varepsilon>0$ and a $\phi \in E$, fix $\mu>w$ and $\phi_{1} \in C^{\beta}([-r, 0], X)$ with

$$
\sup _{-r \leq \xi \leq 0}\|\mu R(\mu, A) \phi(\xi)-\phi(\xi)\| \leq \varepsilon \quad \text { and } \quad\left\|\phi-\phi_{1}\right\|_{E} \leq \varepsilon .
$$

Set $g(s):=\mu R(\mu, A(s)) \phi_{1} \in F_{1, \beta}^{s}$ for $s \geq 0$. Note that

$$
\sup _{s \geq 0}\|g(s)\|_{F_{1, \beta}}<\infty \quad \text { and } \quad \lim _{s \rightarrow \infty}\|g(s)-\phi\|_{E} \leq(K+1) \varepsilon
$$

since $R(\mu, A(s))$ converges strongly to $R(\mu, A)$ as $s \rightarrow \infty$ due to (H1) and the Trotter-Kato theorem. Finally (3.11) and (3.10) lead to

$$
\begin{aligned}
& \varlimsup_{\lim _{s \rightarrow \infty}}\|V(s+t, s) \phi-S(t) \phi\|_{E} \\
& \leq \varlimsup_{s \rightarrow \infty}\left(\|V(s+t, s)(\phi-g(s))\|_{E}\right. \\
& \left.\quad \quad+\|V(s+t, s) g(s)-S(t) g(s)\|_{E}+\|S(t)(g(s)-\phi)\|_{E}\right) \\
& \leq c \varepsilon+\varlimsup_{s \rightarrow \infty}\|V(s+t, s) g(s)-S(t) g(s)\|_{E} \leq c \varepsilon
\end{aligned}
$$

for a constant $c$ independent of $\varepsilon, s$, and $t \in\left[0, t_{0}\right]$. Hence, $V(s+t, s) \rightarrow S(t)$ and, by Proposition [2.1, $P_{V}(s) \rightarrow P_{S}$ strongly on $E$ and uniformly in $t \in\left[0, t_{0}\right]$ as $s \rightarrow \infty$.

Remark 3.7. If $V(\cdot, \cdot)$ is exponentially stable, then we can take $a=0$ in the above theorem. More generally, this is true if $\operatorname{dim} Q_{S} E$ is finite, (3.12) holds, and the adjoint $U(a, 0)^{*}$ is injective on $Q^{*}(a) E^{*}$. This follows from Lemmas 2.4, 2.5, and Remark 2.6 of [21] and Proposition 3.8 below.

Under stronger assumptions we can also show that $V(t, s)$ inherits the dimension of the unstable subspace of $S(t)$. In view of [3, Ex. IV.2.6.3] and [37, §4], condition (3.12) is true for second order elliptic operators on $L^{p}$-spaces with Neumann boundary conditions and sufficiently regular data (provided that $\beta \leq 1 / 2$ ).

Proposition 3.8. Let (H1), (H2), and $\sigma(B) \cap i \mathbb{R}=\emptyset$ hold. We further assume that

$$
\begin{aligned}
& R(w, A(t)) \rightarrow R(w, A) \text { in } \mathcal{L}\left(X, X_{\alpha}^{A}\right) \text { as } t \rightarrow \infty \text { and } \\
& X_{\theta}^{t} \cong X_{\theta}^{A}, t \geq 0, \text { with uniformly equivalent norms }
\end{aligned}
$$

for some $\alpha \in(0,1]$ and $\theta=\beta \vee(1-\alpha)$. Then $\operatorname{dim} Q_{V}(t) E=\operatorname{dim} Q_{S} E$ for $t \geq a$, where $Q_{V}(t), Q_{S}$, and a are given as in Theorem 3.6. 
Proof. (1) The proofs of Theorem 3.6 and Lemma 3.1 show that there is an $a_{1}$ such that $V_{a}(\cdot, \cdot)$ is hyperbolic with uniform constants $N, \delta>0$ for $a \geq a_{1}$, where $V_{a}(\cdot, \cdot)$ solves (2.9) for $L_{a}(\cdot)$ and $U_{a}(\cdot, \cdot)$. Assumption (3.12) yields that $F_{\theta, \beta}^{t} \cong F_{\theta, \beta}^{A}$ for $t \geq 0$ and $\theta=\beta \vee(1-\alpha)$. Thus,

$$
c(t):=\sup _{a \geq a_{1}, s \in \mathbb{R}}\left\|V_{a}(s+t, s)\right\|_{\mathcal{L}\left(E, F_{\theta, \beta}^{A}\right)}<\infty
$$

by Lemma 3.5 if $t \geq 2 r+1=: t_{0}$ so that, setting $Q_{a}(t):=Q_{V_{a}}(t)$,

$$
\begin{aligned}
\sup _{a \geq a_{1}, s \in \mathbb{R}}\left\|Q_{a}(s)\right\|_{\mathcal{L}\left(E, F_{\theta, \beta}^{A}\right)} & =\sup _{a \geq a_{1}, s \in \mathbb{R}}\left\|V_{a}\left(s, s-t_{0}\right) V_{a, Q}\left(s-t_{0}, s\right) Q_{a}(s)\right\|_{\mathcal{L}\left(E, F_{\theta, \beta}^{A}\right)} \\
& \leq c\left(t_{0}\right) N e^{-\delta t_{0}} .
\end{aligned}
$$

In the same way we see that $Q_{S} \in \mathcal{L}\left(E, F_{\theta, \beta}^{A}\right)$. Similarly as (3.10) one derives

$$
\lim _{a \rightarrow \infty} \sup _{s \in \mathbb{R}}\left\|V_{a}(s+t, s)-S(t)\right\|_{\mathcal{L}\left(F_{\theta, \beta}^{A}, E\right)}=0 .
$$

We now use (2.14) to deduce

$$
\begin{gathered}
\left(Q_{S}-Q_{a}(\cdot)\right) Q_{a}(\cdot)=\frac{1}{2 \pi i} \int_{\mathbb{T}} R\left(\lambda, T_{S}(1)\right)\left(T_{V_{a}}(1)-T_{S}(1)\right) Q_{a}(\cdot) R\left(\lambda, T_{V_{a}}(1)\right) d \lambda, \\
\left(Q_{S}-Q_{a}(\cdot)\right) Q_{S}=\frac{1}{2 \pi i} \int_{\mathbb{T}} R\left(\lambda, T_{V_{a}}(1)\right)\left(T_{V_{a}}(1)-T_{S}(1)\right) Q_{S} R\left(\lambda, T_{S}(1)\right) d \lambda .
\end{gathered}
$$

Due to the uniformity of $N$ and $\delta$, the norms of $R\left(\lambda, T_{V_{a}}(1)\right)$ are independent of $a$ (see the proof of [31, Prop. 3.3]). Combining all these facts, we arrive at

$$
\lim _{a \rightarrow \infty} \sup _{t \in \mathbb{R}}\left\|\left(Q_{S}-Q_{a}(t)\right) Q_{S}\right\|=\lim _{a \rightarrow \infty} \sup _{t \in \mathbb{R}}\left\|\left(Q_{S}-Q_{a}(t)\right) Q_{a}(t)\right\|=0 .
$$

(2) Recall that a projection $P$ has the resolvent $R(\lambda, P)=\lambda^{-1}\left(1+(\lambda-1)^{-1} P\right)$ for $\lambda \neq 0,1$. Therefore $R\left(\lambda, Q_{a}(t)\right)$ is uniformly bounded for $a \geq a_{1}$ and $|\lambda-1|=1 / 2$ since $\left\|Q_{a}(t)\right\| \leq N$. Due to (3.13) and (the proof of) [20, Thm. IV.3.16], there is a number $a_{2} \geq a_{1}$ such that $\sigma\left(Q_{S} Q_{a}(t)\right), a \geq a_{2}$, has an open and closed subset in the disc $|\lambda-1|<1 / 2$ and the corresponding spectral subspace $E_{1}$ has the dimension $\operatorname{dim} Q_{a}(t) E$. Since $\left(Q_{S} Q_{a}(t)\right) \mid E_{1}$ is invertible, this identity leads to

$$
\operatorname{dim} Q_{a}(t) E=\operatorname{dim} Q_{S} Q_{a}(t) E_{1} \leq \operatorname{dim} Q_{S} Q_{a}(t) E \leq \operatorname{dim} Q_{S} E .
$$

Analogously one shows the converse inequality so that

$$
\operatorname{dim} Q_{a}(t) E=\operatorname{dim} Q_{S} E
$$

for $a \geq a_{3} \geq a_{2}$ and $t \in \mathbb{R}$. Because of property (b) in the definition of exponential dichotomy it remains to verify that

$$
\operatorname{dim} Q_{a}(t) E=\operatorname{dim} Q_{a_{1}}(t) E
$$

for some $t \in \mathbb{R}$, where we have fixed $a \geq a_{3} \geq a_{1}$. Observe that the stable subspaces are given by

$$
P_{a}(s) E=\left\{\phi \in E: \lim _{t \rightarrow \infty} V(t, s) \phi=0\right\}, \quad s \geq a
$$

(see e.g. [29, p. 551]), and thus coincide with $P_{a_{1}}(s) E$. As a consequence,

$$
\begin{aligned}
& P_{a}(t)=P_{a}(t)\left(P_{a_{1}}(t)+Q_{a_{1}}(t)\right)=P_{a_{1}}(t)+P_{a}(t) V(t, a) V_{a_{1} Q}(a, t) Q_{a_{1}}(t), \\
& \left\|Q_{a}(t)-Q_{a_{1}}(t)\right\| \leq N^{2} e^{-2 \delta(t-a)}<1
\end{aligned}
$$

for large $t \geq a$. The assertion (3.14) now follows from (the arguments in) [20, $\S$ I.4.6]. 


\section{Convergence of mild solutions to the inhomogenous Problem}

We now turn our attention to the inhomogeneous problem (2.11) assuming that $f(t) \rightarrow f_{\infty}$ as $t \rightarrow \infty$. We want to show that

$$
\lim _{t \rightarrow \infty} u(t)=-\left(A+L_{0}\right)^{-1} f_{\infty}=: u_{\infty}
$$

for the mild solution $u$ of (2.11). To that purpose we do not need to restrict ourselves to the parabolic case (H1). So we only assume that $L(\cdot) \in C_{b}\left(\mathbb{R}, \mathcal{L}_{s}(X, E)\right)$ and $U(\cdot, \cdot)$ is an evolution family on $X$ with $J=\mathbb{R}$ satisfying (2.6) with $\omega \geq 0$ (in order to simplify several estimates). Let $V(\cdot, \cdot)$ be the evolution family on $E$ solving (2.9).

We derive in Corollary 4.2 and Proposition 4.3 new 'variation of parameters formulas' expressing the mild solution in terms of $V(\cdot, \cdot)$ and $f$. Here the main difficulty comes from the fact that $f$ maps into $X$ whereas $V(t, s)$ acts on $E$. To deal with this problem, we set

$$
\left(E_{\lambda} f\right)(t, \xi):=e^{\lambda \xi} \int_{-\infty}^{t+\xi} U_{\lambda}(t+\xi, \tau) f(\tau) d \tau, \quad t \in \mathbb{R}, \xi \in[-r, 0],
$$

for $\lambda>\omega$ and $f \in L^{\infty}(\mathbb{R}, X)$; cf. [15, (3.4)]. Clearly, $E_{\lambda} f \in C_{b}(\mathbb{R}, E)$ and

$$
\left\|E_{\lambda} f\right\|_{\infty} \leq \frac{M}{\lambda-\omega}\|f\|_{\infty}
$$

We first show the convergence as $\lambda \rightarrow \infty$ of the function

$$
w_{\lambda}(t)=w_{\lambda}(t ; s, f):=\int_{s}^{t} V(t, \tau) \lambda\left(E_{\lambda} f\right)(\tau) d \tau, \quad t \geq s .
$$

Proposition 4.1. Let $U(\cdot, \cdot)$ be an exponentially bounded evolution family on $X$ with $J=\mathbb{R}$ and $L(\cdot) \in C_{b}\left(\mathbb{R}, \mathcal{L}_{s}(E, X)\right)$. Define $w_{\lambda}$ as in (4.2) for $f \in L^{\infty}(\mathbb{R}, X)$, $t \geq s$, and $\lambda>\omega$. Then $w_{\lambda}(t)$ converges in $E$ to $w(t)=w(t ; s, f)$ as $\lambda \rightarrow \infty$ uniformly for $0 \leq t-s \leq t_{0}$ and $\|f\|_{\infty} \leq c$. Moreover,

$$
w(t ; s, f)=\int_{s}^{(t+\cdot) \vee s} U(t+\cdot, \tau)(L(\tau) w(\tau ; s, f)+f(\tau)) d \tau \quad \text { for } t \geq s .
$$

Proof. Let $t \geq s, f \in L^{\infty}(\mathbb{R}, X)$, and $\lambda, \mu>\omega$. The identities (4.2) and (2.9) yield

$$
\begin{aligned}
w_{\lambda}(t)= & \int_{s}^{t} \tilde{U}(t, \tau) \lambda\left(E_{\lambda} f\right)(\tau) d \tau \\
& \quad+\int_{s}^{t} \int_{\tau}^{(t+\cdot) \vee \tau} U(t+\cdot, \sigma) L(\sigma) V(\sigma, \tau) \lambda\left(E_{\lambda} f\right)(\tau) d \sigma d \tau \\
= & : I_{1}+I_{2} .
\end{aligned}
$$

Notice that

$$
\left[\tilde{U}(t, \tau) \lambda\left(E_{\lambda} f\right)(\tau)\right](\xi)= \begin{cases}\lambda e^{\lambda(t+\xi-\tau)} \int_{-\infty}^{t+\xi} U_{\lambda}(t+\xi, \sigma) f(\sigma) d \sigma, & t+\xi \leq \tau \\ \lambda e^{\lambda(t+\xi-\tau)} \int_{-\infty}^{\tau} U_{\lambda}(t+\xi, \sigma) f(\sigma) d \sigma, & t+\xi>\tau\end{cases}
$$


by (2.8). First, let $t+\xi \geq s$. Integrating by parts, we can transform $I_{1}$ into

$$
\begin{aligned}
I_{1}(\xi)= & \int_{s}^{t+\xi} \lambda e^{\lambda(t+\xi-\tau)} \int_{-\infty}^{\tau} U_{\lambda}(t+\xi, \sigma) f(\sigma) d \sigma d \tau \\
& \quad+\int_{t+\xi}^{t} \lambda e^{\lambda(t+\xi-\tau)} d \tau \int_{-\infty}^{t+\xi} U_{\lambda}(t+\xi, \sigma) f(\sigma) d \sigma \\
= & \int_{s}^{t+\xi} e^{\lambda(t+\xi-\tau)} U_{\lambda}(t+\xi, \tau) f(\tau) d \tau-\int_{-\infty}^{t+\xi} U_{\lambda}(t+\xi, \sigma) f(\sigma) d \sigma \\
& \quad+e^{\lambda(t+\xi-s)} \int_{-\infty}^{s} U_{\lambda}(t+\xi, \sigma) f(\sigma) d \sigma+\left(1-e^{\lambda \xi}\right) \int_{-\infty}^{t+\xi} U_{\lambda}(t+\xi, \sigma) f(\sigma) d \sigma \\
= & \int_{s}^{t+\xi} U(t+\xi, \tau) f(\tau) d \tau+U(t+\xi, s) \int_{-\infty}^{s} U_{\lambda}(s, \tau) f(\tau) d \tau \\
& \quad-\int_{-\infty}^{t+\xi} e^{-\lambda(t-\sigma)} U(t+\xi, \sigma) f(\sigma) d \sigma .
\end{aligned}
$$

Fubini's theorem further implies

$$
\begin{aligned}
I_{2}(\xi) & =\int_{s}^{t+\xi} \int_{s}^{\sigma} U(t+\xi, \sigma) L(\sigma) V(\sigma, \tau) \lambda\left(E_{\lambda} f\right)(\tau) d \tau d \sigma \\
& =\int_{s}^{t+\xi} U(t+\xi, \sigma) L(\sigma) w_{\lambda}(\sigma) d \sigma .
\end{aligned}
$$

Similarly, we obtain

$$
\left[w_{\lambda}(t)\right](\xi)=\left(1-e^{\lambda \xi}\right) \int_{-\infty}^{t+\xi} U_{\lambda}(t+\xi, \sigma) f(\sigma) d \sigma
$$

for $t+\xi \leq s$. Combining (4.4)- (4.7), we estimate

$$
\begin{gathered}
\left\|w_{\lambda}(t)-w_{\mu}(t)\right\|_{E} \leq 2 M e^{\omega(t-s)}\left(\frac{1}{\lambda-\omega}+\frac{1}{\mu-\omega}\right)\|f\|_{\infty} \\
+M\|L(\cdot)\|_{\infty} \int_{s}^{t} e^{\omega(t-\sigma)}\left\|w_{\lambda}(\sigma)-w_{\mu}(\sigma)\right\|_{E} d \sigma, \\
\left\|w_{\lambda}(t)-w_{\mu}(t)\right\|_{E} \leq c e^{(\omega+d)(t-s)}\left(\frac{1}{\lambda-\omega}+\frac{1}{\mu-\omega}\right)\|f\|_{\infty}
\end{gathered}
$$

using Gronwall's inequality, where $c$ and $d$ do not depend on $\mu, \lambda, t, s$. Hence $w_{\lambda}$ tends to a function $w$ as asserted. The identity (4.3) follows from (4.4)-(4.7) by letting $\lambda \rightarrow \infty$.

We now define, for $t \geq s, \phi \in E$, and $f \in L^{\infty}(\mathbb{R}, X)$,

$$
\begin{aligned}
v(t ; s, f)=v(t) & :=V(t, s) \phi+\lim _{\lambda \rightarrow \infty} \int_{s}^{t} V(t, \tau) \lambda\left(E_{\lambda} f\right)(\tau) d \tau \\
& =V(t, s) \phi+w(t ; s, f) \\
u(t) & := \begin{cases}{[v(t)](0),} & t \geq s, \\
\phi(t-s), & s-r \leq t \leq s .\end{cases}
\end{aligned}
$$


The formulas (2.9), (4.3), and (4.8) yield

$$
v(t)=\tilde{U}(t, s) \phi+\int_{s}^{(t+\cdot) \vee s} U(t+\cdot, \tau)(L(\tau) v(\tau)+f(\tau)) d \tau .
$$

This identity shows that $v(t)=u_{t}$ and, hence, that $u$ solves (2.11).

Corollary 4.2. Under the above assumptions, (4.8) and (4.9) yield the unique mild solution $u$ of (2.11), and $u_{t}=v(t)$.

We note that there are other formulas for mild solutions using 'fundamental solutions' or 'resolvent operators'; see [10, 25, 34, 36, Chap. 4], and the references therein. These authors consider different situations partially obtaining stronger results. We have chosen our approach in view of the techniques employed in the proof of Theorem 4.4

In a second step we want to replace in the above formulas the integrals over $[s, t]$ by integrals over $\mathbb{R}$. To that purpose we assume that $V(\cdot, \cdot)$ has an exponential dichotomy with constants $N, \delta>0$, projections $P_{V}(t)$, and Green's function $\Gamma_{V}(\cdot, \cdot)$. We then introduce

$$
\tilde{w}_{\lambda}(t)=\tilde{w}_{\lambda}(t ; f):=\int_{\mathbb{R}} \Gamma_{V}(t, \tau) \lambda\left(E_{\lambda} f\right)(\tau) d \tau
$$

for $t \in \mathbb{R}, \lambda>\omega$, and $f \in L^{\infty}(\mathbb{R}, X)$. Observe that $\tilde{w}_{\lambda}$ is continuous and

$$
\left\|\tilde{w}_{\lambda}(t ; f)\right\| \leq 2 N M(1+\omega) \delta^{-1}\|f\|_{\infty}, \quad \lambda \geq \omega+1, t \in \mathbb{R}
$$

by (4.1). Using a standard argument (see e.g. 7, p. 108]), we deduce from the definitions (4.11) and (4.2) that

$$
\begin{aligned}
\tilde{w}_{\lambda}(t ; f) & =V(t, s) \tilde{w}_{\lambda}(s ; f)+\int_{s}^{t} V(t, \tau) \lambda\left(E_{\lambda} f\right)(\tau) d \tau \\
& =V(t, s) \tilde{w}_{\lambda}(s ; f)+w_{\lambda}(t ; s, f)
\end{aligned}
$$

for $t \geq s$. This expression is the key to the next result.

Proposition 4.3. Let $U(\cdot, \cdot)$ be an exponentially bounded evolution family on $X$ with $J=\mathbb{R}$ and $L(\cdot) \in C_{b}\left(\mathbb{R}, \mathcal{L}_{s}(E, X)\right)$. Assume that the evolution family $V(\cdot, \cdot)$ solving (2.9) on $E$ has an exponential dichotomy. Define $\tilde{w}_{\lambda}$ as in (4.11) for $f \in$ $L^{\infty}(\mathbb{R}, X)$ and $\lambda>\omega$. Then $\tilde{w}_{\lambda}$ converges in $C_{b}(\mathbb{R}, E)$ to a function $\tilde{w}=\tilde{w}(\cdot ; f)$ as $\lambda \rightarrow \infty$ uniformly for $\|f\|_{\infty} \leq c$. Moreover,

$$
\tilde{w}(t ; f)=V(t, s) \tilde{w}(s ; f)+w(t ; s, f), \quad t \geq s,
$$

and the mild solution of (2.11) is given by $u(t)=[v(t)](0)$ and

$$
v(t)=V(t, s)(\phi-\tilde{w}(s ; f))+\tilde{w}(t ; f)
$$

for $t \geq s, \phi \in E$, and $f \in L^{\infty}(\mathbb{R}, X)$.

Proof. Formula (4.13) with $s$ replaced by $t-s$, (4.12), and the exponential dichotomy of $V(\cdot, \cdot)$ imply

$$
\left\|P_{V}(t)\left(\tilde{w}_{\lambda}(t)-\tilde{w}_{\mu}(t)\right)\right\|_{E} \leq c e^{-\delta s}\|f\|_{\infty}+N\left\|w_{\lambda}(t ; t-s, f)-w_{\mu}(t ; t-s, f)\right\|_{E}
$$

for $\lambda, \mu \geq \omega+1, t \in \mathbb{R}, s \geq 0$, and a constant $c \geq 0$. Fix $\varepsilon>0$ and choose $s \geq 0$ such that $c e^{-\delta s}\|f\|_{\infty} \leq \varepsilon$. Proposition 4.1 then shows that

$$
\varlimsup_{\lambda, \mu \rightarrow \infty} \sup _{t \in \mathbb{R}}\left\|P_{V}(t)\left(\tilde{w}_{\lambda}(t)-\tilde{w}_{\mu}(t)\right)\right\|_{E} \leq \varepsilon .
$$


As a result, $P_{V}(\cdot) \tilde{w}_{\lambda}$ converges in $C_{b}(\mathbb{R}, E)$ as $\lambda \rightarrow \infty$. In a similar way we deduce from (4.13) with $s$ replaced by $t$ and $t$ replaced by $s+t$ that

$$
\begin{aligned}
& \quad \begin{array}{l}
V_{Q}(t, t+s) Q_{V}(t+s)\left[\tilde{w}_{\lambda}(t+s)-\tilde{w}_{\mu}(t+s)\right] \\
=Q_{V}(t)\left[\tilde{w}_{\lambda}(t)-\tilde{w}_{\mu}(t)\right] \\
\quad+V_{Q}(t, t+s) Q_{V}(t+s)\left[w_{\lambda}(t+s ; t, f)-w_{\mu}(t+s ; t, f)\right],
\end{array} \\
& \begin{array}{l}
\left\|Q_{V}(t)\left(\tilde{w}_{\lambda}(t)-\tilde{w}_{\mu}(t)\right)\right\|_{E} \\
\quad \leq c e^{-\delta s}\|f\|_{\infty}+N e^{-\delta s}\left\|w_{\lambda}(t+s ; t, f)-w_{\mu}(t+s ; t, f)\right\| .
\end{array}
\end{aligned}
$$

As above we see that $Q_{V}(\cdot) \tilde{w}_{\lambda}$ converges in $C_{b}(\mathbb{R}, E)$ as $\lambda \rightarrow \infty$. The identity (4.14) then follows from (4.13) and Proposition 4.1 The last assertion is a consequence of Corollary 4.2, (4.8), and (4.14).

We are now in a position to establish the convergence of $v(t)$ as $t \rightarrow \infty$. Let $s \in \mathbb{R}$ and $f \in L^{\infty}(\mathbb{R}, X)$ with support in $[s, \infty)$. Because of (4.15) and the boundedness of $\tilde{w}$, the function $v$ is bounded if and only if $\phi-\tilde{w}(s ; f) \in P_{V}(s) E$ if and only if

$$
Q_{V}(s) \phi=\tilde{w}(s ; f)=-\lim _{\lambda \rightarrow \infty} \int_{s}^{\infty} V_{Q}(s, \tau) \lambda\left(E_{\lambda} f\right)(\tau) d \tau .
$$

If this is the case, then

$$
v(t)=V(t, s) P_{V}(s) \phi+\tilde{w}(t ; f), \quad t \geq s .
$$

Theorem 4.4. Let $U(\cdot, \cdot)$ be an exponentially bounded evolution family on $X$ with $J=\mathbb{R}$ and $L(\cdot) \in C_{b}\left(\mathbb{R}, \mathcal{L}_{s}(E, X)\right)$. Take $s \in \mathbb{R}, \phi \in E$, and $f \in L^{\infty}(\mathbb{R}, X)$ with support in $[s, \infty)$ such that (4.16) holds and $f(t) \rightarrow f_{\infty}$ in $X$ as $t \rightarrow \infty$. Assume that the evolution family $V(\cdot, \cdot)$ solving (2.9) on $E$ has an exponential dichotomy with projections $P_{V}(t)$. Let $A$ generate a $C_{0}$-semigroup $T(\cdot)$ on $X, L \in \mathcal{L}(X, E)$, and $S(\cdot)$ be the $C_{0}$-semigroup on $E$ generated by $B$ (see (1.9)). Suppose further that $S(\cdot)$ has an exponential dichotomy with projection $P_{S}$. Finally, assume that $U(\tau+t, \tau) \rightarrow T(t)$ as $\tau \rightarrow \infty$ strongly on $X$ for $t \geq 0$ and that $V(\tau+t, \tau) \rightarrow S(t)$ and $P_{V}(\tau) \rightarrow P_{S}$ as $\tau \rightarrow \infty$ strongly on $E$ for $t \geq 0$. Then the function $v$ given by (4.8) converges in $E$ to $v_{\infty}(\xi)=u_{\infty}=-\left(A+L_{0}\right)^{-1} f_{\infty}, \xi \in[-r, 0]$, and the mild solution $u(t)=[v(t)](0)$ of (2.11) tends to $u_{\infty}$ in $X$ as $t \rightarrow \infty$.

Proof. Proposition 4.3 implies that

$$
\lim _{t \rightarrow \infty} \tilde{w}(t)=\lim _{t \rightarrow \infty} \lim _{\lambda \rightarrow \infty} \tilde{w}_{\lambda}(t)=\lim _{\lambda \rightarrow \infty} \lim _{t \rightarrow \infty} \tilde{w}_{\lambda}(t)
$$

if the double limit on the right hand side exists. Because of (4.17) and the exponential dichotomy of $V(\cdot, \cdot)$ we thus have to show that the limits

$$
\lim _{t \rightarrow \infty} \tilde{w}_{\lambda}(t)=: w_{\lambda, \infty} \quad \text { and } \quad \lim _{\lambda \rightarrow \infty} w_{\lambda, \infty} \equiv-\left(A+L_{0}\right)^{-1} f_{\infty}
$$


exist in $E$. We first write

$$
\begin{aligned}
\tilde{w}_{\lambda}(t)=\int_{0}^{\infty} V(t, t-\tau) P_{V}(t-\tau) \lambda e^{\lambda \cdot} \int_{-\infty}^{t-\tau+\cdot} U_{\lambda}(t-\tau+\cdot \sigma) f(\sigma) d \sigma d \tau \\
\quad-\int_{0}^{\infty} V_{Q}(t, t+\tau) Q_{V}(t+\tau) \lambda e^{\lambda \cdot} \int_{-\infty}^{t+\tau+\cdot} U_{\lambda}(t+\tau+\cdot, \sigma) f(\sigma) d \sigma d \tau \\
=\int_{0}^{\infty} V(t, t-\tau) P_{V}(t-\tau) \lambda e^{\lambda \cdot} \\
\quad \times \int_{0}^{\infty} U_{\lambda}(t-\tau+\cdot, t-\tau+\cdot-\sigma) f(t-\tau+\cdot-\sigma) d \sigma d \tau \\
-\int_{0}^{\infty} V_{Q}(t, t+\tau) Q_{V}(t+\tau) \lambda e^{\lambda} . \\
\quad \times \int_{0}^{\infty} U_{\lambda}(t+\tau+\cdot, t+\tau+\cdot-\sigma) f(t+\tau+\cdot-\sigma) d \sigma d \tau
\end{aligned}
$$

We may assume that $T(\cdot)$ also satisfies (2.6). Fix $\varepsilon \in(0,1), \tau \in \mathbb{R}$, and $\xi \in[-r, 0]$. For $t \in \mathbb{R}, \lambda \geq \omega+1$, and $t_{0}>0$, we further compute

$$
\begin{gathered}
D_{\mp}(t, \tau, \xi):=\int_{0}^{\infty}\left[U_{\lambda}(t \mp \tau+\xi, t \mp \tau+\xi-\sigma) f(t \mp \tau+\xi-\sigma)-e^{-\lambda \sigma} T(\sigma) f_{\infty}\right] d \sigma \\
=\int_{0}^{\infty} U_{\lambda}(t \mp \tau+\xi, t \mp \tau+\xi-\sigma)\left(f(t \mp \tau+\xi-\sigma)-f_{\infty}\right) d \sigma \\
\quad+\int_{0}^{\infty} e^{-\lambda \sigma}[U(t \mp \tau+\xi, t \mp \tau+\xi-\sigma)-T(\sigma)] f_{\infty} d \sigma \\
\left\|D_{\mp}(t, \tau, \xi)\right\| \leq \int_{0}^{t_{0}} M e^{(\omega-\lambda) \sigma}\left\|f(t \mp \tau+\xi-\sigma)-f_{\infty}\right\| d \sigma \\
\quad+\int_{t_{0}}^{\infty} 4 M e^{(\omega-\lambda) \sigma}\|f\|_{\infty} d \sigma \\
\quad+\int_{0}^{t_{0}} e^{-\lambda \sigma}\left\|[U(t \mp \tau+\xi, t \mp \tau+\xi-\sigma)-T(\sigma)] f_{\infty}\right\| d \sigma \\
\leq M \sup _{0 \leq \sigma \leq t_{0}}\left\|f(t \mp \tau+\cdot-\sigma)-f_{\infty}\right\|_{E}+4 M e^{-t_{0}}\|f\|_{\infty} \\
\quad+\int_{0}^{t_{0}}\left\|[U(t \mp \tau+\cdot t \mp \tau-\sigma+\cdot)-T(\sigma)] f_{\infty}\right\|_{E} d \sigma .
\end{gathered}
$$

Fixing a large $t_{0}$ and letting $t \rightarrow \infty$, this estimate leads to

$$
\varlimsup_{t \rightarrow \infty} \sup _{\xi \in[-r, 0]}\left\|D_{\mp}(t, \tau, \xi)\right\| \leq \varepsilon
$$

for each $\tau \in \mathbb{R}$ due to the assumptions and the theorem of dominated convergence. Therefore the inner integrals in (4.20) converge in $E$ to

$$
\int_{0}^{\infty} e^{-\lambda \sigma} T(\sigma) f_{\infty} d \sigma=R(\lambda, A) f_{\infty}
$$

as $t \rightarrow \infty$ for each $\tau \in \mathbb{R}$. By assumption, $V(t, t-\tau) P_{V}(t-\tau)$ tends strongly to $S(\tau) P_{S}$ as $t \rightarrow \infty$ for each $\tau \in \mathbb{R}$. One easily sees that

$$
V_{Q}(t, t+\tau) Q_{V}(t+\tau) \rightarrow S_{Q}(-\tau) Q_{S}
$$


strongly (compare with part (1) of the proof of [32, Thm. 4.1]). Moreover, the outer integrands in (4.20) are bounded by $c e^{-\delta \tau}$ for $\lambda \geq \omega+1$. As a result of Lebesgue's convergence theorem and [11, Exer. V.1.19], we thus obtain

$$
\begin{aligned}
\lim _{t \rightarrow \infty} \tilde{w}_{\lambda}(t) & =\int_{0}^{\infty} S(\tau) P_{S} \lambda e^{\lambda \cdot} R(\lambda, A) f_{\infty} d \tau-\int_{0}^{\infty} S_{Q}(-\tau) Q_{S} \lambda e^{\lambda \cdot} R(\lambda, A) f_{\infty} d \tau \\
& =-B^{-1} \lambda e^{\lambda \cdot} R(\lambda, A) f_{\infty}=: w_{\lambda, \infty} .
\end{aligned}
$$

Hence, $w_{\lambda, \infty} \in D(B)$ and $w_{\lambda, \infty}^{\prime}(\xi)=B w_{\lambda, \infty}(\xi)=-\lambda e^{\lambda \xi} R(\lambda, A) f_{\infty}$, which yields

$$
w_{\lambda, \infty}(\xi)=x_{\lambda}+\left(1-e^{\lambda \xi}\right) R(\lambda, A) f_{\infty}
$$

for some $x_{\lambda}=w_{\lambda, \infty}(0) \in D(A)$. Moreover, $w_{\lambda, \infty}^{\prime}(0)=A x_{\lambda}+L w_{\lambda, \infty}$ so that

$$
-\lambda R(\lambda, A) f_{\infty}=A x_{\lambda}+L_{0} x_{\lambda}+L\left(1-e^{\lambda \cdot}\right) R(\lambda, A) f_{\infty} .
$$

Since $S(\cdot)$ is hyperbolic, $A+L_{0}$ is invertible by [11, Thm. IV.3.6, Prop. VI.6.7]. Thus,

$$
x_{\lambda}=-\left(A+L_{0}\right)^{-1}\left[\lambda R(\lambda, A) f_{\infty}+L\left(1-e^{\lambda \cdot}\right) R(\lambda, A) f_{\infty}\right] \longrightarrow-\left(A+L_{0}\right)^{-1} f_{\infty}
$$

as $\lambda \rightarrow \infty$. This fact combined with (4.21) leads to the second identity in (4.19).

\section{The inhomogeneous problem in the parabolic Case}

We assume again that (H1), (H2), and $\sigma(B) \cap i \mathbb{R}=\emptyset$ hold. Fix $a$ as obtained in Theorem 3.6. We study the problem

$$
\begin{aligned}
& u(t)=U(t, a) \phi(0)+\int_{a}^{t} U(t, \tau)\left(L(\tau) u_{\tau}+f(\tau)\right) d \tau, \quad t \geq a, \\
& u(t)=\phi(t-a), \quad a-r \leq t \leq a,
\end{aligned}
$$

for $\phi \in E$ and $f \in C_{b}([a, \infty), X)$ converging to $f_{\infty}$ in $X$ as $t \rightarrow \infty$. Because of Lemma 3.4 and Theorem 3.6, we can apply Theorem 4.4 to conclude that

$$
\exists u_{\infty}:=\lim _{t \rightarrow \infty} u(t)=-\left(A+L_{0}\right)^{-1} f_{\infty}
$$

if and only if (4.16) with $s=a$ holds. (Here we extend $f$ by 0 to a function on $\mathbb{R}$ and use the hyperbolic evolution family solving (2.9) for $U_{a}(\cdot, \cdot)$ and $L_{a}(\cdot)$ as in the proof of Theorem 3.6.) To obtain the classical solutions of (1.1), we have to require more regularity for $L(\cdot)$ and $f$. Here we use the space

$$
E_{\theta}^{t}:=\left\{\phi \in E:\|\phi\|_{E_{\theta}^{t}}:=\sup _{\xi \in[-r, 0]}\|\phi(\xi)\|_{\theta}^{t+\xi}<\infty\right\}
$$

endowed with the norm $\|\cdot\|_{E_{\theta}^{t}}$ and the assumptions

(H3) $f \in C_{b}^{\gamma}\left(\mathbb{R}_{+}, X\right)$ and $L(\cdot) \in C_{b}^{\gamma}\left(\mathbb{R}_{+}, \mathcal{L}(E, X)\right)$ for some $\gamma \in(0,1)$;

$\left(\mathrm{H}^{\prime}\right) f \in C_{b}\left(\mathbb{R}_{+}, X\right)$, and $\|f(t)\|_{\gamma}^{t}$ and $L(t): E_{\gamma}^{t} \rightarrow X_{\gamma}^{t}$ are uniformly bounded for $t \geq 0$ and some $\gamma \in(0,1)$.

We now come to the second main result of this paper extending [32, Thm. 4.1] where the undelayed case was treated.

Theorem 5.1. Assume that (H1), (H2), $\sigma(B) \cap i \mathbb{R}=\emptyset$, and either (H3) or (H3') hold. Fix $a \geq 0$ as obtained in Theorem 3.6. Let $\phi \in E$ satisfy (4.16) with $s=a$ and $f(t) \rightarrow f_{\infty}$ in $X$ as $t \rightarrow \infty$. Then the mild solution $u$ of (5.1) is continuously differentiable on $[a+2 r+2, \infty), u(t) \in D(A(t))$, and

$$
\dot{u}(t)=A(t) u(t)+L(t) u_{t}+f(t) \quad \text { for } \quad t \geq a+2 r+2 .
$$


Moreover, u fulfills (5.2),

$$
\lim _{t \rightarrow \infty} \dot{u}(t)=0, \quad \text { and } \quad \lim _{t \rightarrow \infty} A(t) u(t)=A u_{\infty} .
$$

Proof. We have already proved (5.2). Assume for a moment that $u$ satisfies (5.3) and that $\dot{u}(t)$ tends to 0 as $t \rightarrow \infty$. Then

$$
\lim _{t \rightarrow \infty} A(t) u(t)=-\lim _{t \rightarrow \infty}\left(L(t) u_{t}+f(t)\right)=-L_{0} u_{\infty}-f_{\infty}=A u_{\infty} .
$$

Thus the second part of (5.4) follows from the first one and (5.3). Extend $f$ by 0 to $\mathbb{R}$ and recall that $u(t)=[v(t)](0)$ and

$$
\begin{aligned}
v(t)= & V(t, a) P_{V}(a) \phi+\tilde{w}(t ; f) \\
= & V(t, a) P_{V}(a) \phi+V(t, s) \tilde{w}(s ; f) \\
& \quad+\int_{s}^{t+\cdot} U(t+\cdot, \tau)(L(\tau) w(\tau ; s, f)+f(\tau)) d \tau \\
& =: S_{1}(t)+S_{2}(t, s)+S_{3}(t, s)
\end{aligned}
$$

for $t \geq s+r$ and $s \geq a$ by (4.9), (4.17), (4.14), and (4.3). We first assume that (H3) holds and establish the differentiability of $V(\cdot, s) \psi$ in step (1). The derivatives of $S_{k}(t)$ are estimated in step (2). The required regularity of $u$ and (5.3) also follow from (1) and (2). Then we perform (more briefly) the analogous steps $\left(1^{\prime}\right)$ and $\left(2^{\prime}\right)$ supposing $\left(\mathrm{H} 3^{\prime}\right)$.

(1) We verify that $V(\cdot, s) \psi$ is Hölder continuous in $E$ for $\psi \in E$. Let $s \geq a$ and $s+r+1 \leq t^{\prime} \leq t \leq t^{\prime}+t_{0}$. Using (2.9) we calculate

$$
\begin{aligned}
(V(t, s) \psi)(\xi)-\left(V\left(t^{\prime}, s\right) \psi\right)(\xi) \\
=\left[U\left(t+\xi, t^{\prime}+\xi\right)-1\right]\left(U\left(t^{\prime}+\xi, s\right) \psi(0)+\int_{s}^{t^{\prime}+\xi} U\left(t^{\prime}+\xi, \tau\right) L(\tau) V(\tau, s) \psi d \tau\right) \\
\quad+\int_{t^{\prime}+\xi}^{t+\xi} U(t+\xi, \tau) L(\tau) V(\tau, s) \psi d \tau \\
=\left[U\left(t+\xi, t^{\prime}+\xi\right)-1\right]\left(U\left(t^{\prime}+\xi, t^{\prime}+\xi-1\right)\left(V\left(t^{\prime}-1, s\right) \psi\right)(\xi)\right. \\
\left.\quad+\int_{t^{\prime}+\xi-1}^{t^{\prime}+\xi} U\left(t^{\prime}+\xi, \tau\right) L(\tau) V(\tau, s) \psi d \tau\right)+\int_{t^{\prime}+\xi}^{t+\xi} U(t+\xi, \tau) L(\tau) V(\tau, s) \psi d \tau
\end{aligned}
$$

for $\xi \in[-r, 0]$. Therefore (2.3) and (2.1) allow us to estimate

$$
\begin{aligned}
& \left\|V(t, s) \psi-V\left(t^{\prime}, s\right) \psi\right\|_{E} \\
& \leq c\left(t-t^{\prime}\right)^{\theta}\left(\left\|V\left(t^{\prime}-1, s\right) \psi\right\|_{E}+\sup _{-r \leq \xi \leq 0} \int_{t^{\prime}+\xi-1}^{t^{\prime}+\xi}\left(t^{\prime}+\xi-\tau\right)^{-\theta}\|V(\tau, s) \psi\|_{E} d \tau\right) \\
& \quad+c\left(t-t^{\prime}\right) \sup _{t^{\prime}-r \leq \tau \leq t}\|V(\tau, s) \psi\|_{E} \\
& \leq c\left(t-t^{\prime}\right)^{\theta} \sup _{t^{\prime}-r-1 \leq \tau \leq t}\|V(\tau, s) \psi\|_{E}
\end{aligned}
$$


for $\theta \in(0,1)$ and constants $c$ independent of $\psi, t, t^{\prime}, s$ as chosen above. Hence, $L(\cdot) V(\cdot, s) \psi \in C^{\gamma}([s+r+1, \infty), X)$ by $(\mathrm{H} 3)$. We further have

$$
[V(t, s) \psi](\xi)=\left[V\left(t, t_{1}\right) V\left(t_{1}, s\right) \psi\right](\xi)
$$

$$
=U\left(t+\xi, t_{1}\right)\left[V\left(t_{1}, s\right) \psi\right](0)+\int_{t_{1}}^{t+\xi} U(t+\xi, \tau) L(\tau) V(\tau, s) \psi d \tau
$$

for $t>t_{1}+r$ and $t_{1} \geq s$. Thus $t \mapsto V(t, s) \psi$ is continuously differentiable in $E$ and $\partial_{t} V(t, s) \psi(\xi)=A(t+\xi) U\left(t+\xi, t_{1}\right)\left[V\left(t_{1}, s\right) \psi\right](0)+L(t+\xi) V\left(t+\xi, t_{1}\right) V\left(t_{1}, s\right) \psi$

$$
\begin{aligned}
& +A(t+\xi) \int_{t_{1}}^{t+\xi} U(t+\xi, \tau) L(\tau) V(\tau, s) \psi d \tau \\
= & : S_{4}(t, s)(\xi)+S_{5}(t, s)(\xi)+S_{6}(t, s)(\xi)
\end{aligned}
$$

for $t>t_{1}+r \geq s+2 r+1$ and $\xi \in[-r, 0]$ due to [2, Thm. 6.1(i)].

(2) We can now estimate the derivative of the first summand in (5.6) using (5.9) with $\psi=P_{V}(a) \phi, s=a, t \geq a+2 r+2$, and $t_{1}=t-1-r$. By means of (2.1), 2, Thm. 6.1(ii)], (H3), (5.7), and the exponential dichotomy of $V(\cdot, \cdot)$, we obtain

$$
\begin{aligned}
\left\|\frac{d}{d t} S_{1}(t)\right\|_{E} \leq & c\left\|V(t+r-1, a) P_{V}(a) \phi\right\|_{E} \\
& +c\left\|L(\cdot) V(\cdot, a) P_{V}(a) \phi\right\|_{C^{\gamma}([t-1-r, t], X)} \\
\leq & c e^{-\delta(t-a-2 r-2)}\|\phi\|_{E}
\end{aligned}
$$

for $t \geq a+2 r+2$ and constants $c$ independent of $t$ and $\phi$.

For $\frac{d}{d t} \tilde{w}(t)=\partial_{1} S_{2}(t, s)+\partial_{1} S_{3}(t, s)$ we first establish a global Hölder estimate. (Here $\partial_{1}$ denotes the derivative with respect to the first variable.) The equality (4.3), 1, Thm. 4.3], and (4.1) yield

$$
\|w(\cdot ; s, f)\|_{C^{\theta}([s, s+T], E)} \leq c_{T}\left[\|f\|_{\infty}+\|w(\cdot ; s, f)\|_{C([s, s+T], E)}\right] \leq c_{T}^{\prime}
$$

for $\theta \in(0,1)$ and constants independent of $s$. Employing (H3) and [2, Thm. 6.1(i)], we then derive

$$
\left[\partial_{1} S_{3}(t, s)\right](0)=A(t)\left[S_{3}(t, s)\right](0)+L(t) w(t ; s, f)+f(t)
$$

for $t \geq s+r$. Together with (5.6), (5.8), and (5.9), this gives us the asserted regularity of $u(t)$ and

$$
\begin{aligned}
\dot{u}(t) & =A(t) u(t)+L(t)\left[V(t, a) P_{V}(a) \phi+V(t, s) \tilde{w}(s ; f)+w(t ; s, f)\right]+f(t) \\
& =A(t) u(t)+L(t) u_{t}+f(t)
\end{aligned}
$$

for $t>a+2 r+1$, where we have used $v(t)=u_{t}$ in the second equality.

We further deduce from (5.6), [2, Thm. 6.1(ii)], (H3), and (5.11) that

$$
\left\|\partial_{1} S_{3}(t, s)-\partial_{1} S_{3}\left(t^{\prime}, s\right)\right\|_{E} \leq c\left|t-t^{\prime}\right|^{\gamma}
$$

for $s+2 r+3 \geq t, t^{\prime} \geq s+r+1 / 2$ and a constant independent of $s$. Moreover, (5.6) combined with (5.11) and [2, Thm. 6.1(ii)] implies that

$$
\sup _{t \in[s+r, s+2 r+2]}\left\|\partial_{1} S_{3}(t, s)\right\|_{E} \leq c,
$$

where $c$ does not depend on $s$. 
In order to obtain analogous estimates for $\partial_{1} S_{2}(t, s)$, we involve (5.9) with $\psi=$ $\tilde{w}(s ; f)$. Let $s+2 r+3 / 2 \leq t_{1}+r+1 / 2 \leq t^{\prime}, t \leq t_{1}+r+2=: t_{2}$. We estimate

$$
\begin{aligned}
\left\|S_{4}(t, s)-S_{4}\left(t^{\prime}, s\right)\right\|_{E} & \leq c\left|t-t^{\prime}\right|^{\gamma}\left\|V\left(t_{1}, s\right) \tilde{w}(s ; f)\right\|_{E} \\
\left\|S_{5}(t, s)-S_{5}\left(t^{\prime}, s\right)\right\|_{E} & \leq c\left|t-t^{\prime}\right|^{\gamma}\|V(\cdot, s) \tilde{w}(s ; f)\|_{C^{\gamma}\left(\left[t_{1}+1 / 2, t_{2}\right], E\right)} \\
& \leq c\left|t-t^{\prime}\right|^{\gamma} \sup _{\tau \in\left[t_{1}-r-1 / 2, t_{2}\right]}\|V(\tau, s) \tilde{w}(s ; f)\|_{E} \\
\left\|S_{6}(t, s)-S_{6}\left(t^{\prime}, s\right)\right\|_{E} & \leq c\left|t-t^{\prime}\right|^{\gamma}\|L(\cdot) V(\cdot, s) \tilde{w}(s ; f)\|_{C^{\gamma}\left(\left[t_{1}, t_{2}\right], X\right)} \\
& \leq c\left|t-t^{\prime}\right|^{\gamma} \sup _{\tau \in\left[t_{1}-r-1, t_{2}\right]}\|V(\tau, s) \tilde{w}(s ; f)\|_{E}
\end{aligned}
$$

using (2.5), (H3), (5.7), and [2, Thm. 6.1(ii)]. Take $s=t-2-2 r$ and $t_{1}=$ $s+r+1=t-r-1$ in the above estimates and recall that $\tilde{w}$ is uniformly bounded. Then we arrive at

$$
\left\|\partial_{1} S_{2}(t, t-2-2 r)-\partial_{1} S_{2}\left(t^{\prime}, t-2-2 r\right)\right\|_{E} \leq c\left|t-t^{\prime}\right|^{\gamma}
$$

for a constant not depending on the above chosen $t, t^{\prime}$. Similarly one sees that

$$
\sup _{t \geq s+2 r+2}\left\|\partial_{1} S_{2}(t, t-2-2 r)\right\|_{E}<\infty .
$$

Choosing $s=t-2 r-2$ in (5.6), the inequalities (5.13)-(5.16) yield

$$
\frac{d}{d t} \tilde{w} \in C_{b}^{\gamma}([a+2 r+2, \infty), E)
$$

as required. On the other hand, $\tilde{w}(t ; f)$ tends in $E$ to $v_{\infty} \equiv u_{\infty}$ as $t \rightarrow \infty$ by (4.18) and (4.19). Let $\hat{w}(t):=\tilde{w}(t)-v_{\infty}$. The interpolation result 23, Cors. 1.2.19, 0.2 .2 , and (5.17) then imply

$$
\begin{aligned}
\sup _{n \leq t \leq n+1}\left\|\frac{d}{d t} \tilde{w}(t)\right\|_{E} & \leq\left\|\frac{d}{d t} \hat{w}\right\|_{C^{h}([n, n+1], E)} \\
& \leq c\|\hat{w}\|_{C([n, n+1], E)}^{1-\theta}\|\hat{w}\|_{C^{1+\gamma}([n, n+1], E)}^{\theta} \\
& \leq c \sup _{n \leq t \leq n+1}\left\|\tilde{w}(t)-v_{\infty}\right\|_{E}^{1-\theta} \longrightarrow 0 \quad \text { as } n \rightarrow \infty,
\end{aligned}
$$

where $h \in(0, \gamma), \theta=\frac{1+h}{1+\gamma}$, and the constants do not depend on $n \geq a+2 r+2$. Because of (5.5) and (5.10), the assertion is verified in the case that (H3) holds.

(1') Employing (5.8) for $t_{1}=t-r-1 \geq s \geq a$ and (2.1), we derive

$$
\|V(t, s) \psi(\xi)\|_{\gamma}^{t+\xi} \leq c \sup _{\tau \in[t-r-1, t]}\|V(\tau, s) \psi\|_{E}
$$

for $t \geq s+r+1 \geq a+r+1$. The equality (5.9) then follows from (5.8), (5.18), $\left(\mathrm{H} 3^{\prime}\right)$, and [2, Thm. 6.2(i)].

$\left(2^{\prime}\right)$ Take $s=a, t \geq a+2+2 r, t_{1}=t-r-1$, and $\psi=P_{V}(a) \phi$ in (5.9). Then (2.1), (5.18), $\left(\mathrm{H}^{\prime}\right)$, 2, Thm. 6.2(iii)], and the exponential dichotomy of $V(\cdot, \cdot)$ imply that

$$
\left\|\partial_{t} V(t, a) P_{V}(a) \phi\right\|_{E} \leq c e^{-\delta(t-a-2 r-2)}\|\phi\|_{E} .
$$

From (4.3), 1, Thm. 4.3], and (4.1) we further deduce

$$
\|w(t ; s, f)\|_{E_{\theta}^{t}} \leq c_{T}
$$

for $\theta \in(0,1), t \in[s, s+T]$, and a constant independent of $s$. The identity (5.3) for $t \geq a+2 r+2$ is now a consequence of (5.6), (5.9), (5.20), $\left(\mathrm{H} 3^{\prime}\right)$, and [2, Thm. $6.2(\mathrm{i})]$. 
We fix $s=t-2-2 r \geq a$ in (5.6). Then [2, Thm. 6.2(iii)], (H3'), and (5.20) lead to

$$
\sup _{t \geq a+2 r+2}\left\|\partial_{1} S_{3}(t, t-2-2 r)\right\|_{E_{\gamma}^{t}}<\infty .
$$

To deal with $S_{2}$, we involve (5.9) for $\psi=\tilde{w}(s ; f), t_{1}=t-r-1, s=t-2 r-2$, and $t \geq a+2 r+2$. This gives

$$
\begin{aligned}
& \left\|\partial_{1} V(t, t-2 r-2) \tilde{w}(t-2 r-2)\right\|_{E_{\gamma}^{t}} \\
& \quad \leq c \sup _{t-r-1 \leq \tau \leq t}\|V(\tau, t-2 r-2) \tilde{w}(t-2 r-2)\|_{E_{\gamma}^{\tau}} \\
& \quad \leq c \sup _{t-2 r-2 \leq \tau \leq t}\|V(\tau, t-2 r-2) \tilde{w}(t-2 r-2)\|_{E} \\
& \quad \leq c
\end{aligned}
$$

thanks to [32, Prop. 2.4], (5.18), (H3'), and [2, Thm. 6.2(iii)]. Putting (5.21) and (5.22) together, we conclude

$\sup _{t \geq a+2 r+2}\left\|\frac{d}{d t} \tilde{w}(t)\right\|_{E_{\gamma}^{t}}=\sup _{t \geq a+2 r+2}\left\|\partial_{1} S_{2}(t, t-2 r-2)+\partial_{1} S_{3}(t, t-2 r-2)\right\|_{E_{\gamma}^{t}}<\infty$.

We set $y(t):=[\tilde{w}(t)](0)=u(t)-\left[V(t, a) P_{V}(a) \phi\right](0)$ and remark that

$$
\sup _{t \geq a+2 r+2}\|\dot{y}(t)\|_{\gamma}^{t}<\infty .
$$

Because of $\dot{y}(t)=A(t) y(t)+L(t) y_{t}+f(t)$, the assumptions, (5.2), and (5.19) imply

$$
\begin{aligned}
R(w, A(t)) \dot{y}(t) & =-y(t)+w R(w, A(t)) y(t)+R(w, A(t)) L(t) y_{t}+R(w, A(t)) f(t) \\
& \rightarrow-u_{\infty}+w R(w, A) u_{\infty}+R(w, A) L_{0} u_{\infty}+R(w, A) f_{\infty} \\
& =R(w, A)\left[\left(A+L_{0}\right) u_{\infty}+f_{\infty}\right]=0
\end{aligned}
$$

as $t \rightarrow \infty$. On the other hand, the moment inequality (see e.g. [11, Thm. II.5.34]), the embedding [11, Prop. II.5.33], and (5.23) allow us to estimate

$$
\begin{aligned}
\|\dot{y}(t)\| & \leq c\left\|(w-A(t))^{-1} \dot{y}(t)\right\|^{1-\theta}\left\|(w-A(t))^{h} \dot{y}(t)\right\|^{\theta} \\
& \leq c\left\|(w-A(t))^{-1} \dot{y}(t)\right\|^{1-\theta}\left(\|\dot{y}(t)\|_{\gamma}^{t}\right)^{\theta} \\
& \leq c\|R(w, A(t)) \dot{y}(t)\|^{1-\theta}
\end{aligned}
$$

for $h \in(0, \gamma), \theta:=\frac{1}{1+h}$, and constants $c$ independent of $t \geq a+2 r+2$. As a consequence of (5.24) we thus obtain that $\dot{y}(t)$ tends to 0 as $t \rightarrow \infty$. Together with (5.19) this establishes the theorem also under the hypothesis $\left(\mathrm{H} 3^{\prime}\right)$.

The above proof leads to the following regularity result for mild solutions.

Proposition 5.2. Assume that $A(\cdot)$ satisfies $(P)$ and that $L(\cdot) \in C_{b}\left(\mathbb{R}_{+}, \mathcal{L}_{s}(E, X)\right)$ satisfies either (H3) or (H3'). Then the mild solution $u$ of (2.11) is continuously differentiable on $(s+r, \infty), u(t) \in D(A(t))$ for $t>s+r$, and $u$ fulfills (1.1) for $t>s+r$. If in addition $\phi(0) \in D\left(A(s)\right.$ ) and either $\phi \in C^{\gamma}([-r, 0], X)$ (in case (H3)) or $\phi \in E_{\gamma}^{s}$ (in case (H')), then the assertion holds with $t>s+r$ replaced by $t \geq s$.

Proof. The first assertion follows from (4.8), (4.3), (5.8), and the arguments used in parts (1) and $\left(1^{\prime}\right)$ of the proof of Theorem 5.1 Similarly one sees that the function $\tau \mapsto L(\tau) u_{\tau}+f(\tau), \tau \geq s$, is either Hölder continuous or bounded in $X_{\gamma}^{\tau}$ under the 
additional assumption on $\phi$. Thus (2.11) and [2, Thms. 6.1, 6.2] imply the second assertion.

Remark 5.3. Using Lemma 3.5 and modifying the proofs in a straightforward way, one can replace (H3) in Theorem 5.1 and Proposition 5.2 by the condition

(H3a) $f \in C_{b}^{\gamma}\left(\mathbb{R}_{+}, X\right)$ and $L(\cdot) \in C_{b}^{\gamma}\left(\mathbb{R}_{+}, \mathcal{L}\left(C^{h}([-r, 0], X), X\right)\right)$ for some $\gamma, h \in$ $(0,1)$.

Example 5.4. We investigate the retarded heat equation

$$
\begin{aligned}
& \partial_{t} u(t, x)=a(t, x) \partial_{x x} u(t, x)+c(t, x) u(t, x) \\
& \quad-l(t, x) u(t-\tau(t), x)+f(t, x), t \geq 0, x \in[0, \pi], \\
& u(t, 0)=u(t, \pi)=0, \quad t \geq 0 \\
& u(t, x)=\phi(t, x), \quad-2 \leq t \leq 0, x \in[0, \pi]
\end{aligned}
$$

where $a, c, l \in C_{b}^{\mu}\left(\mathbb{R}_{+}, C[0, \pi]\right), \tau \in C^{\mu}\left(\mathbb{R}_{+},[0,2]\right), \mu \in(0,1), a(t, x) \geq a_{0}>0$, and

$$
a(t, x) \rightarrow 1, \quad c(t, x) \rightarrow 1+\varepsilon, \quad l(t, x) \rightarrow \frac{\pi}{2} e^{\varepsilon}, \quad \tau(t) \rightarrow 1
$$

uniformly for $x \in[0, \pi]$ as $t \rightarrow \infty$ for some $\varepsilon \geq 0$. Let $X=L^{2}(0, \pi)$ and $D=$ $W^{2,2}(0, \pi) \cap W_{0}^{1,2}(0, \pi)$ be endowed with the usual norms. Set

$$
A(t) \varphi=a(t, \cdot) \varphi^{\prime \prime}+c(t, \cdot) \varphi \quad \text { and } \quad A f=\varphi^{\prime \prime}+(1+\varepsilon) \varphi
$$

for $\varphi \in D$ and $t \geq 0$. One easily verifies (H1) with $\nu=1$ and (3.12) with $\alpha=\theta=1$ using elliptic regularity. On $E=C([-2,0], X)$ we further define

$$
L(t) \phi=-l(t, \cdot) \phi(-\tau(t)) \text { and } L \phi=-\frac{\pi}{2} e^{\varepsilon} \phi(-1) .
$$

Observe that (H2) is satisfied for each $\beta \in(0,1)$ and that (H3a) holds with $h=$ $\gamma=\mu$ if $f \in C_{b}^{\mu}\left(\mathbb{R}_{+}, X\right)$. (One could also work with space regularity of $l$ and $f$ using $\left(\mathrm{H} 3^{\prime}\right)$.)

Define $B$ as in (1.9). In [36, pp. 78-80] it is shown that $\sigma(B) \cap i \mathbb{R}=\emptyset$ for small $\varepsilon>0$. For $\varepsilon=0$, there are two simple eigenvalues $\pm i \frac{\pi}{2}$ with corresponding eigenfunctions $\phi_{ \pm}$and spectral projection $Q$; the rest of the spectrum has real part smaller than $d<0$.

Thus, for small $\varepsilon>0, V(t, s)$ has an exponential dichotomy on an interval $[a, \infty)$ with two-dimensional unstable subspaces. We obtain the convergence of the mild solution if $f(t) \rightarrow f_{\infty}$ and (4.16) holds with $s=a$. If $\varepsilon=0$, one sees by a rescaling argument that there is a splitting such that $V(t, s) P(s)$ is exponentially stable. For $\phi \in E$ there are numbers $c_{ \pm}$such that $Q \phi=c_{+} \phi_{+}+c_{-} \phi_{-}$. Theorem [3.6] also implies that

$$
V(s+t, s) Q(s) \phi \longrightarrow S(t) Q \phi=c_{+} e^{i t \frac{\pi}{2}} \phi_{+}+c_{-} e^{-i t \frac{\pi}{2}} \phi_{-}
$$

as $s \rightarrow \infty$ for $t \geq 0$ and $\phi \in E$.

\section{REFERENCES}

[1] P. Acquistapace, Evolution operators and strong solutions of abstract linear parabolic equations, Differential Integral Equations 1 (1988), 433-457. MR 90b:34094

[2] P. Acquistapace, B. Terreni, A unified approach to abstract linear nonautonomous parabolic equations, Rend. Sem. Mat. Univ. Padova 78 (1987), 47-107. MR 89e:34099

[3] H. Amann, Linear and Quasilinear Parabolic Problems. Volume 1: Abstract Linear Theory, Birkhäuser, 1995. MR 96g:34088

[4] A. Batkái, Hyperbolicity of linear partial differential equations with delay, Integral Equations Operator Theory 44 (2002), 383-396. MR 2003j:34148 
[5] A. Batkái, R. Schnaubelt, Asymptotic behaviour of parabolic problems with delays in the highest order derivatives, submitted.

[6] C.J.K. Batty, R. Chill, Approximation and asymptotic behaviour of evolution families, Differential Integral Equations 15 (2002), 477-512. MR 2002i:34100

[7] C. Chicone, Y. Latushkin, Evolution Semigroups in Dynamical Systems and Differential Equations, Amer. Math. Soc., 1999. MR 2001e:47068

[8] K.L. Cooke, Linear functional differential equations of asymptotically autonomous type, J. Differential Equations 7 (1970), 154-174. MR 41:604

[9] R. Datko, Not all feedback stabilized systems are robust with respect to small time delays, SIAM J. Control Optim. 26 (1988), 697-713. MR 89c:93057

[10] W. Desch, I. Győri, G. Gühring, Stability of nonautonomous delay equations with a positive fundamental solution, as a preprint in: Tübinger Berichte zur Funktionalanalysis 9 (2000), 125-139.

[11] K.J. Engel, R. Nagel, One-Parameter Semigroups for Linear Evolution Equations, Springer, 2000. MR 2000i: 47075

[12] G. Gühring, Asymptotic properties of nonautonomous evolution equations and nonautonomous retarded equations, Ph.D. thesis, Tübingen, 1999.

[13] G. Gühring, F. Räbiger, Asymptotic properties of mild solutions of nonautonomous evolution equations with applications to retarded differential equations, Abstr. Appl. Anal. 4 (1999), 169-194. MR 2001m:34130

[14] G. Gühring, F. Räbiger, W. Ruess, Principle of linearized stability for semilinear nonautonomous evolution equations with applications to retarded differential equations, Differential Integral Equations 13 (2000), 503-528. MR 2001c:34124

[15] G. Gühring, F. Räbiger, R. Schnaubelt, A characteristic equation for non-autonomous partial functional differential equations, J. Differential Equations 181 (2002), 439-462. MR 2003d:34168

[16] D. Guidetti, On the asymptotic behavior of solutions of linear nonautonomous parabolic equations, Boll. Un. Mat. Ital. B (7) 1 (1987), 1055-1076. MR 89d:35077

[17] I. Győri, F. Hartung, J. Turi, Preservation of stability in delay equations under delay perturbations, J. Math. Anal. Appl. 220 (1998), 290-312. MR 99a:34212

[18] J.K. Hale, Theory of Functional Differential Equations, Springer, 1977. MR 58:22904

[19] J.K. Hale, S.M. Verduyn Lunel, Effects of small delays on stability and control, in: Bart, Gohberg, Ran (Eds.): Operator Theory and Analysis, The M.A. Kaashoek Anniversary Volume, Birkhäuser, 2001, pp. 275-301. MR 2002e:93043

[20] T. Kato, Perturbation Theory for Linear Operators, Corrected Printing of 2nd Edition, Springer, 1980. MR 53:11389

[21] X.-B. Lin, Exponential dichotomies and homoclinic orbits in functional differential equations, J. Differential Equations 63 (1986), 227-254. MR 87j:34138

[22] X.-B. Lin, Exponential dichotomies in intermediate spaces with applications to a diffusively perturbed predator-prey model, J. Differential Equations 108 (1994), 36-63. MR 95c:35139

[23] A. Lunardi, Analytic Semigroups and Optimal Regularity in Parabolic Problems, Birkhäuser, 1995. MR 96e: 47039

[24] J. Mallet-Paret, The Fredholm alternative for functional differential equations of mixed type, J. Dynam. Differential Equations 11 (1999), 1-48. MR 2000j:34107

[25] J. Prüss, On resolvent operators for linear integrodifferential equations of Volterra type, J. Integral Equations 5 (1983), 211-236. MR 85d:45026

[26] A. Rhandi, Extrapolation methods to solve nonautonomous retarded partial differential equations, Studia Math. 126 (1997), 219-233. MR 99c:47058

[27] W.M. Ruess, Existence of solutions to partial functional differential equations with delay, in: A.G. Kartsatos (ed.), Theory and Applications of Nonlinear Operators of Accretive and Monotone Type, Marcel Dekker, 1996, 259-288. MR 97b:34085

[28] R. Schnaubelt, Exponential bounds and hyperbolicity of evolution equations, Ph.D. thesis, Tübingen, 1996.

[29] R. Schnaubelt, Sufficient conditions for exponential stability and dichotomy of evolution equations, Forum Math. 11 (1999), 543-566. MR 2001c:34121

[30] R. Schnaubelt, Exponential dichotomy of nonautonomous evolution equations, Habilitation thesis, Tübingen, 1999. 
[31] R. Schnaubelt, A sufficient condition for exponential dichotomy of parabolic evolution equations, in: G. Lumer, L. Weis (eds.), Evolution Equations and their Applications in Physical and Life Sciences (Proceedings Bad Herrenalb, 1998), Marcel Dekker, 2000. MR 2001m:34132

[32] R. Schnaubelt, Asymptotically autonomous parabolic evolution equations, J. Evol. Equ. 1 (2001), 19-37. MR 2002e:34095

[33] R. Schnaubelt, Asymptotic behaviour of parabolic nonautonomous evolution equations, Report No. 12 (2002), FB Mathematik und Informatik, University of Halle (preprint).

[34] K. Schumacher, On the resolvent of linear nonautonomous partial functional differential equations, J. Differential Equations 59 (1985), 355-387. MR 87b:35164

[35] H. Tanabe, Equations of Evolution, Pitman, 1979. MR 82g:47032

[36] J. Wu, Theory and Applications of Partial Functional Differential Equations, Springer, 1996. MR 98a:35135

[37] A. Yagi, Parabolic equations in which the coefficients are generators of infinitely differentiable semigroups II, Funkcial. Ekvac. 33 (1990), 139-150. MR 91h:47039

[38] A. Yagi, Abstract quasilinear evolution equations of parabolic type in Banach spaces, Boll. Un. Mat. Ital. B (7) 5 (1991), 351-368. MR 92h:47100

FB Mathematik und Informatik, Martin-Luther-Universität, 06099 Halle, Germany

E-mail address: schnaubelt@mathematik.uni-halle.de 